\title{
JGR Oceans
}

\section{RESEARCH ARTICLE}

10.1029/2021JC017570

Key Points:

- An empirical model using seasonal harmonics is developed to characterize internal tide variability

- Seasonal variations in standing internal tides generated from multiple sources lead to temporal modulations of individual harmonics

- Internal tide predictability at a site is dependent on standing wave node locations

Correspondence to:

M. D. Rayson,

matt.rayson@uwa.edu.au

Citation:

Rayson, M. D., Jones, N. L., Ivey, G. N., \& Gong, Y. (2021). A seasonal harmonic model for internal tide amplitude prediction. Journal of Geophysical Research: Oceans, 126, e2021JC017570. https://doi.org/10.1029/2021JC017570

Received 14 MAY 2021

Accepted 24 SEP 2021

\section{A Seasonal Harmonic Model for Internal Tide Amplitude Prediction}

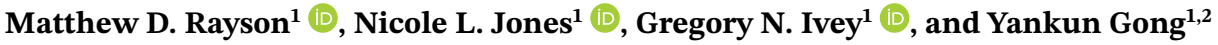 \\ ${ }^{1}$ Oceans Graduate School and the Oceans Institute, University of Western Australia, Crawley, WA, Australia, ${ }^{2}$ State \\ Key Laboratory of Tropical Oceanography, South China Sea Institute of Oceanology, Chinese Academy of Sciences, \\ Guangzhou, China
}

\begin{abstract}
We present an empirical model of the seasonal variability of the internal tide using seasonal harmonics to modulate the amplitude of the fundamental tidal constituents. Internal tide data, from both long-term, in-situ moorings and a mesoscale-resolving and internal tide-resolving ocean model, are used to demonstrate the performance of the seasonal harmonic model for the Indo-Australian Basin region. The seasonal model describes up to $15 \%$ more of the observed (baroclinic) sea surface height variance than a fixed-amplitude harmonic model at the mooring sites. The ocean model results demonstrate that the study region, which includes the Australian North West Shelf (NWS), Timor Sea, and southern Indonesian Islands, is dominated by standing wave interference patterns due to the presence of multiple generation sites. The seasonal harmonic model reveals that temporal shifts in the standing wave patterns coincide with seasonal variations in density stratification. This shift is particularly evident within distances of 2-3 internal wave lengths from strong generation sites. The fraction of the variance of the internal tide signal explained by seasonal modulations is largest in standing wave node regions, contributing to differences in predictive skill of the seasonal harmonic model at two moorings separated by only $38 \mathrm{~km}$. Output of the harmonic model also demonstrates that the seasonally evolving $M_{2}$ internal tide propagating southward from Lombok Strait had a small amplitude in October when shear from the Indonesian Throughflow was strongest.
\end{abstract}

Plain Language Summary Internal waves drive variability in ocean properties such as sea surface height or internal water temperature. In some regions, most of this temporal variability is centered around the tidal frequencies, i.e., oscillating once or twice per day, due to the surface tides generating the waves. Surface tides are readily predictable using a technique called harmonic analysis due to the mechanical response of the ocean mass to the gravitational pull from the Sun and Moon. While internal waves are forced by these surface tides, they are also influenced by temporally variable ocean conditions such as the ocean density. Here, we modify the standard fixed harmonic analysis method to account for seasonal variations in ocean properties. For some applications, internal wave-induced variability is considered to be noise and therefore deterministic methods for describing this variability (the noise) are needed.

\section{Introduction}

Prediction of internal tides-internal waves of tidal frequency-is important for numerous practical applications. For example, accurate prediction of internal tides is a crucial step in interpreting the future Surface Water Ocean Topography (SWOT) high-resolution altimetry mission, and hence in obtaining the submesoscale variability (e.g., Arbic et al., 2015; Ray \& Zaron, 2011). There is, however, temporal variability at seasonal scales in internal tides as seen, for example, in the observational findings on the Australian North West Shelf (NWS) and Timor Sea reported by Rayson et al. (2012) and Kelly et al. (2014), respectively. In this paper, we aim to understand the spatial extent of these seasonal variations on the resulting baroclinic velocity and isotherm displacement fields in the NWS and Timor Sea region. More broadly, we are also motivated by the global internal tide-resolving model analyses of Shriver et al. (2014) and Nelson et al. (2019) who demonstrated that the nonstationary component of the signal (defined below) can comprise a significant portion of the total variance in some locations.
(C) 2021. American Geophysical Union. All Rights Reserved. 
Internal tide prediction techniques largely originate from surface tide methods, namely harmonic analysis. Prediction of surface tides is either through empirical harmonic models with fixed tidal frequencies and spatially varying harmonic amplitudes or response-based models (e.g., Foreman, 1977; Munk \& Cartwright, 1966). The harmonic amplitudes are estimated from either tide gauge data, satellite altimetry sea surface height data, or from solutions to the shallow-water equations (Egbert \& Ray, 2017). A key characteristic of sites that are not predictable using this approach is that, instead of single spectral peaks, their spectral content exhibits broad "cusps" around each of the forcing frequencies (Colosi \& Munk, 2006; Munk $\&$ Cartwright, 1966). Broad spectral cusps are also found in surface tide records where the tides undergo modulations due to low-frequency water level variations (e.g., from storm surge), changing bathymetry, or nonlinear effects due to drag.

For internal tide observations, broad spectral cusps centered around the fundamental tidal frequencies determined by the barotropic tide seem to be the rule rather than the exception (e.g., Colosi \& Munk, 2006; Van Haren, 2004). The frequency smearing is caused by intermittency in wave arrival due to a combination of processes including: temporal variations in stratification and mesoscale flow (Buijsman et al., 2017; Ponte \& Klein, 2015; Rainville \& Pinkel, 2006; Zaron \& Egbert, 2014), time-varying bottom stratification, and incoming internal wave interference that cause variations in topographic generation (Gong et al., 2019; Kelly \& Nash, 2010). Given that they do not form sharp spectral peaks, internal tides are broadly defined as the band-passed portion of the signal of an ocean variable like baroclinic velocity or buoyancy perturbation (Buijsman et al., 2017; Nash, Kelly, et al., 2012).

It is common (within some of the internal tide literature) to name the portion of the band-passed signal that can be determined using discrete tidal harmonics as the "coherent" internal tide, and the residual as the "incoherent" internal tide (e.g., Dushaw et al., 2011; Kelly \& Nash, 2010; Nash, Shroyer, et al., 2012; Pickering et al., 2015; Van Haren, 2004). Other internal tide literature, predominantly produced by the satellite altimetry and numerical model communities, use the terms "stationary" and "nonstationary" to label the harmonically deterministic and nondeterministic components, respectively (e.g., Arbic et al., 2015; Savage et al., 2017; Zaron, 2017). Nonstationary is usually defined as the signal variance changing in time, rather than another statistical property, such as the signal mean. However, as has previously been mentioned (e.g., Nash, Shroyer, et al., 2012), and as we will highlight below, the aforementioned decomposition into stationary/nonstationary, or coherent/incoherent, components is dependent on the choice of frequencies and the record length used for the harmonic analysis, i.e., the choice of the deterministic function. Here, we show that the deterministic seasonal internal wave amplitude variability is important, and that some of this variability has likely been interpreted as a nonstationary portion of the signal in previous studies (e.g., Nelson et al., 2019).

Zaron (2019) used the approach of Huess and Andersen (2001) to account for seasonal variations in the $M_{2}$ baroclinic sea surface height amplitude by including an annual modulation, where the seasonal component is represented in the additional harmonics $M A_{2}$ and $M B_{2}$, which have a frequency offset by one cycle per year from $M_{2}$. In Section 2, we extend this approach and demonstrate that seasonal variations in the amplitude of the major astronomical tidal frequencies $\left(M_{2}, K_{1}\right.$, etc.) lead to new spectral peaks that are offset by integer multiples of the annual frequency. These are the "seasonal sidelines" of the internal tide as hinted at by Arbic et al. (2015). These discrete, seasonal spectral peaks have previously been identified in the surface tide literature (e.g., Cartwright \& Tayler, 1971; Doodson, 1921) so the seasonal model described below is by no means novel. Rather than being directly forced by astronomical frequencies like the surface tide, our interpretation is that internal tide signals can be modeled using a small number of discrete frequencies that are modulated in time.

The Australian North West Shelf (NWS), Timor Sea, and Indonesian Archipelago are regions where large-amplitude internal tides emanating from different generation sites interact (Bachman et al., 2020; Gong et al., 2019; Holloway, 2001; Kelly et al., 2014; Rayson et al., 2012). Nash, Shroyer, et al. (2012) in their assessment of 16 shelf mooring locations around the globe, found through harmonic analysis that the most predictable site was in the Timor Sea (ITFTIS site in Figure 1). However, Kelly et al. (2014) showed (by fitting harmonics to 30 days segment lengths of baroclinic velocity) that the $M_{2}$ tidal harmonic at this site underwent annual modulations. They theorized that changes in seasonal stratification, coherent on the length scale of the wave propagation distance of a few hundred kilometers at this site, were responsible for 


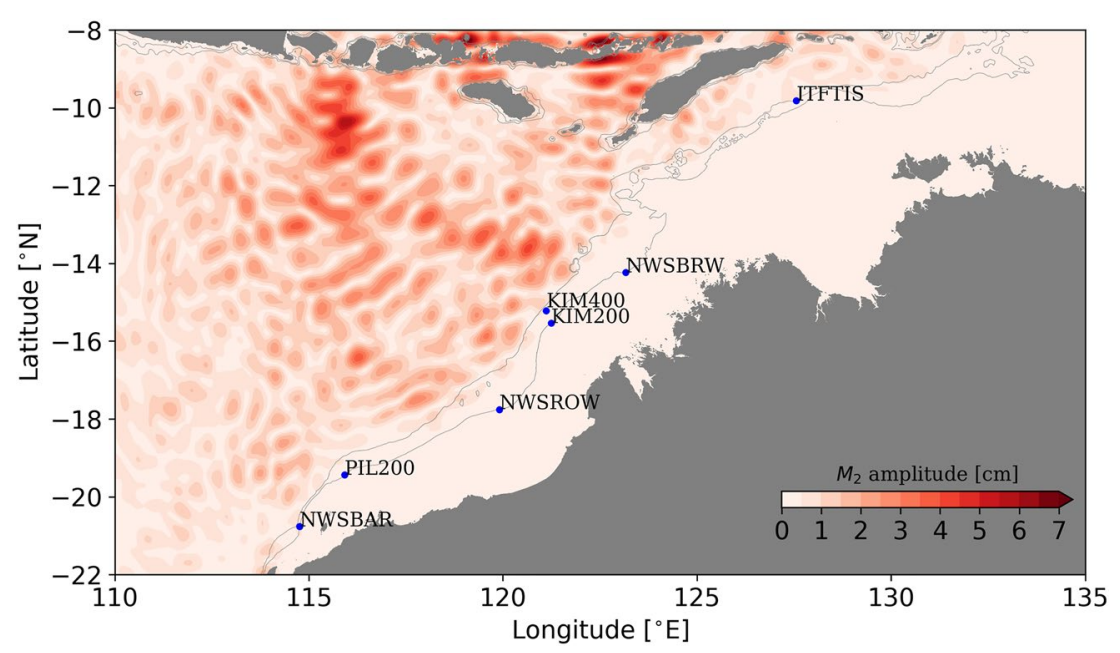

Figure 1. Map of the field sites with the $M_{2}$ baroclinic sea surface height amplitude (cm) from Zaron (2019) overlaid. Gray lines indicate the 200-m and 500-m depth contours that highlight the edge of the continental shelf.

the annual modulation of the internal tide. Rayson et al. (2012) used observations from a mooring on the Kimberley section of the NWS to show that the amplitude of semidiurnal velocity and buoyancy perturbations underwent seasonal modulations. They used a 2D analytical model to demonstrate that the observed signal response was the result of seasonal variations in stratification that, in turn, led to changes in the position of the nodes and antinodes of the standing internal tide.

Standing internal tides with seasonal shifts have been reported in other regions e.g., the Hawaiian Ridge (Rainville et al., 2010), the South China Sea (Ray \& Zaron, 2011), and the Bay of Bengal (Jithin et al., 2020). Zaron (2019) reported the global distribution of seasonally modulated $M_{2}$ baroclinic sea surface height variance ( $M A_{2}$ and $M B_{2}$ constituents) derived from satellite altimetry. The Western Pacific and Amazon shelf were highlighted in the paper although the Indonesian Archipelago, the Coral Sea, and Western Indian Ocean were also regions with a strong seasonal component.

The structure of this paper is as follows. In Section 2, we present the seasonal harmonic model and define several metrics for characterizing the seasonality of internal tides. Descriptions of the in-situ data and numerical model setup are given in Section 3. Section 4 begins with an overview of the in-situ observations before a quantitative evaluation of the seasonal harmonic model is presented. In Section 5, we present a regional overview of the internal tide seasonality using the primitive equation ocean model solution, and explore potential physical drivers in Section 6. We conclude with an overview of the potential uses of an internal tide climatology data set and suggest potential modifications to the harmonic model.

\section{Seasonal Harmonic Model}

Variations in tidally forced quantities such as the internal wave amplitude, $a$, are typically modeled using tidal harmonics by employing a series of sinusoidal basis functions with fixed frequencies and amplitudes (cf. Egbert \& Ray, 2017; Foreman, 1977)

$$
a_{i}=\sum_{m} \alpha_{m} \cos \left(\omega_{m} t_{i}\right)+\beta_{m} \sin \left(\omega_{m} t_{i}\right)+\varepsilon
$$

where $\omega_{m}$ are the tidal harmonic frequencies [cycles $\mathrm{d}^{-1}$ ], $t_{i}$ is the time in days at step $i, \varepsilon$ is a residual term, and $\alpha_{m}$ and $\beta_{m}$ are fixed amplitudes for each harmonic, $m$. Best estimates of these fixed-amplitude parameters are typically found by linear least-squares fitting to time series observations of $a_{i}$. Note that the 18.6 years lunar nodal modulation is known to vary the amplitude of the major astronomical tidal constituents, so Equation 1 is only relevant to short-time series (roughly 1 year).

In contrast, a time-dependent harmonic amplitude model is

$$
a_{i}=\sum_{m} \alpha_{m, i} \cos \left(\omega_{m} t_{i}\right)+\beta_{m, i} \sin \left(\omega_{m} t_{i}\right)+\varepsilon_{a}
$$


where the key difference between Equations 1 and 2 is that the amplitudes $\alpha_{m, i}$ and $\beta_{m, i}$, and hence the signal variance, now vary with time. This approach results in more unknown variables than data points, however, and hence the amplitude modulation must be parameterized. Several observational (Nash, Shroyer, et al., 2012; Rayson et al., 2012) and modeling studies (Buijsman et al., 2017; Savage et al., 2017; Shriver et al., 2014) have demonstrated that harmonic fitting to internal tide signals with four or five major astronomical forcing frequencies using only short record lengths (30 days or less) results in good predictions (i.e., roughly $>80 \%$ of the variance explained). A general approach to predicting the time-dependent internal tide amplitude is then to create a time series model of the amplitudes from these short-time harmonic fits; i.e., a model that captures the $M_{2}$ amplitude variations from one 30-day time window to the next. Shriver et al. (2014) utilized a similar approach where they fit a single annual harmonic to amplitudes from overlapping 30-day harmonic fits, using several years of a global internal tide-resolving model output.

Our seasonal harmonic model allows the amplitudes of the major astronomical forcing frequencies to vary slowly in time by using $N$ seasonal harmonics. With the annual frequency $\omega_{A}=2 \pi / 365.25 \mathrm{~d}^{-1}$, the real and imaginary amplitudes are now

$$
\alpha_{m, i}=\widehat{\alpha}_{m, 0}+\sum_{n=1}^{N} \widehat{\alpha}_{m, n} \cos \left(n \omega_{A} t_{i}\right)+\widehat{\beta}_{m, n} \sin \left(n \omega_{A} t_{i}\right)
$$

and

$$
\beta_{m, i}=\tilde{\alpha}_{m, 0}+\sum_{n=1}^{N} \tilde{\alpha}_{m, n} \cos \left(n \omega_{A} t_{i}\right)+\tilde{\beta}_{m, n} \sin \left(n \omega_{A} t_{i}\right),
$$

respectively. The complex time-varying amplitude for any tidal constituent, $m$, is

$$
\hat{\eta}_{m, i}=\alpha_{m, i}+i \beta_{m, i},
$$

where $i=\sqrt{-1}$, and Equation 5 will be used throughout this paper to describe the internal tide amplitude variability and to relate it back to physical processes.

In principle, it is possible to estimate the unknown parameters in Equations 2-4 using linear least-squares methods in two steps. In the first step, short-time harmonic fits are used to estimate $\alpha_{m, i}$ and $\beta_{m, i}$ for discrete window periods and, in the second step, the seasonal harmonic amplitudes (parameters $\hat{\alpha} \widehat{\beta}$, $\tilde{\alpha}$, and $\tilde{\beta}$ ) are least-squares fit to the amplitudes calculated in step one. The down side of this approach is that one must arbitrarily define a suitable window length. Alternatively, Equations 2-4 can be combined to give

$$
a_{t}=\sum_{m} \sum_{n=-N}^{N} A_{m, j} \cos \left(\left[\omega_{m}+n \omega_{A}\right] t_{i}\right)+B_{m, j} \sin \left(\left[\omega_{m}+n \omega_{A}\right] t_{i}\right)+\varepsilon_{a},
$$

where the subscript $j=n+N+1$. Equation 6 demonstrates that direct incorporation of the seasonal variation results in additional discrete bands offset by $\pm n \omega_{A}$ around the major astronomical forcing frequencies $\omega_{m}$. Note that some of these frequencies correspond to the major astronomical tidal constituents, e.g., $P_{1}=K_{1}-2 \omega_{A}$ and $K_{2}=S_{2}+2 \omega_{A}$ (see Doodson, 1921). However, most of the seasonal sideline frequencies in the internal tide arise from a nonlinear response to the changing propagation medium. We will demonstrate this point below with a numerical model that is forced with eight discrete frequencies, but results in significant energy in these seasonally created spectral bands distributed around the forcing frequencies.

The unknown parameters, which must be estimated from the observed data, are the amplitude matrices $A_{m, j}$ and $B_{m, j}$ that have $M$ rows and $2 N+1$ columns. For example, below we use five tidal constituents $(M=5)$ and three annual harmonics $(N=3)$, so $A_{m, j}$ and $B_{m, j}$ each have 35 elements. The practical benefit of writing the seasonal harmonic model in the form of Equation 6 is that the parameters can be estimated in one step using linear leastsquares fitting. Temporal modulation of the real and imaginary amplitudes of each tidal harmonic (the terms in Equations 3 and 4) are then back-calculated from the amplitude matrices in Equation 6 according to

$$
\begin{gathered}
\hat{\alpha}_{m, 0}=A_{m, N+1} \\
\tilde{\alpha}_{m, 0}=B_{m, N+1} \\
\hat{\alpha}_{m, n}=A_{m, N-n+1}+A_{m, N+n+1} \\
\hat{\beta}_{m, n}=B_{m, N+n+1}-B_{m, N-n+1} \\
\tilde{\alpha}_{m, n}=B_{m, N-n+1}+B_{m, N+n+1} \\
\tilde{\beta}_{m, n}=A_{m, N-n+1}-A_{m, N+n+1} .
\end{gathered}
$$


We use these amplitudes to then obtain $\eta_{m, i}$ from Equation 5 in order to investigate the temporal variations in the major astronomical tidal harmonics (e.g., $M_{2}, S_{2}, N_{2}, K_{1}, O_{1}$ ).

Diverse metrics have been used in the literature to quantify the nonstationarity of internal tides. Shriver et al. (2014) computed tidal fits to 18330 days segments from 9 years of global HyCOM (numerical model) SSH data. They use the normalized RMS of the amplitude for all 183 time blocks as a metric for nonstationarity. In their discussion, they also fit annual harmonics to the amplitudes (their Figure 11). Nash, Shroyer, et al. (2012) used incoherence as a metric for nonstationarity. Their definition for coherence was determined by the percentage of variance in the 6-30 $\mathrm{h}$ band-pass filtered baroclinic current records which could be explained by fitting 8 tidal harmonics to 90 days segments. Ray and Zaron (2011) fit tidal harmonics to altimetry data using data from specific months to identify seasonality, and hence nonstationarity, of the tidal harmonics.

Although our model is stationary, in the sense that the total variance is constant in time, the amplitudes of the major astronomical tidal constituents do vary in time. We characterize this temporal variability of the major tidal constituents, along with the overall performance of the seasonal model, using four metrics. The fraction of the total variance explained by the seasonal harmonic fit (SHVF) is

$$
S H V F=\frac{1}{2} \frac{\sum_{m=1}^{5} \sum_{j=1}^{2 N+1}\left|\widehat{A}_{m, j}\right|^{2}}{\left\langle S S H_{B C}\right\rangle^{2}},
$$

where $\hat{A}_{m, j}=A_{m, j}+i B_{m, j}$ is a complex matrix, and $\left\langle S S H_{B C}\right\rangle^{2}$ is the total signal variance. A similar definition also applies to the tidal harmonic model, which we call THVF

$$
T H V F=\frac{1}{2} \frac{\sum_{m=1}^{5}\left|\widehat{A}_{m, N+1}\right|^{2}}{\left\langle S S H_{B C}\right\rangle^{2}} .
$$

These metrics define the performance of the harmonic model fit and are equivalent to calculating a Murphy Skill score (Murphy, 1988). The variance around an individual astronomical tidal harmonic, including its annual harmonics, is

$$
V F_{m}=\frac{\sum_{j=1}^{2 N+1}\left|\widehat{A}_{m, j}\right|^{2}}{\sum_{m=1}^{5} \sum_{j=1}^{2 N+1}\left|\widehat{A}_{m, j}\right|^{2}} .
$$

The last metric we calculate is the variance fraction of the seasonal harmonics relative to the major astronomical tidal constituent, defined as

$$
S V F_{m}=1-\frac{\left|\widehat{A}_{m, N+1}\right|^{2}}{\sum_{j=1}^{2 N+1}\left|\widehat{A}_{m, j}\right|^{2}} .
$$

The metric $V F_{m}$ estimates the importance of a particular harmonic to the total internal tide signal, while $S V F_{m}$ estimates the importance of seasonal modulation to that particular harmonic.

\section{Methods}

\subsection{Baroclinic Sea Surface Height Estimation}

We focused our analyses on the baroclinic sea surface height perturbation $S S H_{B C}$ because it is an integrated metric of the water column response to the passage of internal waves. Furthermore, it can be derived from satellite altimetry observations and is therefore often the quantity of interest for regional and global internal tide studies (e.g., Gong et al., 2021; Nelson et al., 2019; Savage et al., 2017; Shriver et al., 2014; Zaron, 2019; Zhao et al., 2016). We also performed analyses on the buoyancy mode amplitude but chose to not include this as the seasonal variability was qualitatively similar to $S S H_{B C}$.

Two terminologies for $S_{S H}$ exist in the literature: surface baroclinic pressure (see e.g., Kelly, 2016; Wunsch, 2013; Zhao et al., 2016), and steric height (see e.g., Savage et al., 2017). The hydrostatic approx- 
Table 1

Details of Each In-Situ Mooring Used in This Study to Measure Through-Water-Column Temperature

\begin{tabular}{llllc}
\hline Site ID & \multicolumn{1}{c}{ Location } & Water depth $(\mathrm{m})$ & \multicolumn{1}{c}{ Deployment period } & No. Instruments \\
\hline ITFTIS & Timor Sea & 460 & 2010-2019 & 17 \\
KIM200 & Kimberley & 200 & March 2012 to August 2014 & 14 \\
KIM400 & Kimberley & 405 & March 2012 to August 2014 & 17 \\
PIL200 & Pilbara & 202 & March 2012 to August 2014 & 14 \\
NWSBAR & Barrow Island & 200 & August 2019 to February 2020 & 15 \\
NWSROW & Rowley Shoals & 200 & August 2019 to February 2020 & 14 \\
NWSBRW & Browse Island & 200 & August 2019 to February 2020 & 15 \\
\hline
\end{tabular}

imation and simplified assumptions about the surface and bottom boundary conditions of pressure (see Wunsch, 2013) are made to estimate $S S H_{B C}$ from internal motions. Here, we use the surface pressure definition of Zhao et al. (2016) where

$$
S S H_{B C}=\frac{p_{\text {surf }}}{\rho_{0} g}
$$

and

$$
p_{\text {surf }}=\rho_{0} \int_{-H}^{0} b d z
$$

is the surface baroclinic pressure perturbation, $\rho_{0}=1,024 \mathrm{~kg} \mathrm{~m}^{-3}$ is a constant reference density, $H$ is the water depth, and $b$ is the buoyancy perturbation resulting from density $\rho$ being perturbed about some background density $\langle\rho\rangle$ i.e.,

$$
b=-\frac{(\rho-\langle\rho\rangle) g}{\rho_{0}} .
$$

The surface pressure results from the requirement that the depth-integrated baroclinic pressure must be zero (Kunze et al., 2002), although this assumes there is negligible heaving of the background density field by either the free-surface or barotropic flow over topography (see Kelly et al., 2010, for details).

\subsection{In-Situ Mooring Data}

Multiyear time series of internal tide-induced sea surface height perturbation $S S H_{B C}$ were inferred from water temperature observations from vertical moorings deployed in water depths greater than $200 \mathrm{~m}$ along the outer region of the Australian North West Shelf and Timor Sea (Figure 1). Moorings were deployed as part of the Australian Integrated Marine Observing System (IMOS) between 2010 and 2020, with servicing conducted roughly every 6 months (see Table 1 for deployment periods at each site). Each mooring was equipped with Seabird 37/39/56 thermistors that measured water temperature at 60-s intervals. Instruments were nominally spaced at 20-m depth increments with the uppermost thermistor located $20-30 \mathrm{~m}$ below mean sea level (Table 1). Data from an additional three IMOS moorings that collected through-water-column temperature data from August 2019 to February 2020 were also used as validation data.

We used Equation 12 to infer the sea surface height perturbation from mooring data by first converting temperature to density using a nonlinear equation of state with the climatological mean salinity at each site. The density was extrapolated to the surface and seabed by using the value from the closest thermistor, which were typically located about $20 \mathrm{~m}$ below the free-surface and 1-2 $\mathrm{m}$ above the seabed, respectively. Instead of using the raw observed density in Equation 12, we used the band-passed filtered density (third-order Butterworth with 6 and $60 \mathrm{~h}$ cutoff periods) to compute $S S H_{B C}$. The justification for a $6 \mathrm{~h}$ cutoff period is that buoyancy perturbations with shorter periods are more likely to be nonhydrostatic whereas the definition of $\mathrm{SSH}_{B C}$ in Equation 12 invokes the hydrostatic approximation. 


\subsection{SUNTANS Model}

A realistic three-dimensional primitive equation ocean solver was used to model the basin-scale ocean circulation, with tides, for a 12-month period. The purpose of the ocean model was to capture the seasonal variations in large-scale circulation, stratification, and their influence on the tidally generated internal waves. The Indo-Australian Basin and the surrounding shelf seas and island chains were investigated in detail; it is one example of many global regions where large-scale flow is likely to influence temporal variability of internal tides, which propagate from many different topographic generation regions (Gong et al., 2021; Rayson et al., 2012).

\subsubsection{Governing Equations}

We employed the hydrostatic version of the unstructured grid Stanford University Nonhydrostatic Terrain-following Adaptive Navier-Stokes (SUNTANS) solver (Fringer et al., 2006) to model the ocean circulation. The model solves the Reynolds-averaged Navier-Stokes equations with the Boussinesq and hydrostatic approximations

$$
\begin{aligned}
& \frac{\partial u}{\partial t}+\nabla \cdot(\mathbf{u} u)-f v=-g \frac{\partial}{\partial x}(\eta+r)+\nabla_{H} \cdot\left(v_{H} \nabla u\right)+\frac{\partial}{\partial z}\left(v_{v} \frac{\partial u}{\partial z}\right), \\
& \frac{\partial v}{\partial t}+\nabla \cdot(\mathbf{u} v)+f u=-g \frac{\partial}{\partial y}(\eta+r)+\nabla_{H} \cdot\left(v_{H} \nabla v\right)+\frac{\partial}{\partial z}\left(v_{v} \frac{\partial v}{\partial z}\right),
\end{aligned}
$$

where $\nabla=(\partial / \partial x, \partial / \partial y, \partial / \partial z),(u, v, w)$ are the eastward, northward, and vertical velocity components, respectively, $f$ is the Coriolis frequency, and $v_{H}$ and $v_{v}$ are the horizontal and vertical eddy viscosity. The free-surface elevation is $\eta$ and $r$ is the pressure head due to internal density fluctuations given by

$$
r=\frac{1}{\rho_{0}} \int_{z}^{\eta} \rho^{\prime} d z
$$

where $\rho_{0}$ is the reference density $\left(1,000 \mathrm{~kg} \mathrm{~m}^{-3}\right)$, and $\rho^{\prime}=\rho-\rho_{0}$ is a perturbation density. The continuity equation is

$$
\nabla \cdot \mathbf{u}=0,
$$

and the free surface, $\eta$, is updated by solving the depth-integrated continuity equation

$$
\frac{\partial \eta}{\partial t}+\frac{\partial}{\partial x}\left(\int_{-H}^{\eta} u d z\right)+\frac{\partial}{\partial y}\left(\int_{-H}^{\eta} v d z\right)=0
$$

The tracer (temperature and salinity) transport equations are

$$
\begin{gathered}
\frac{\partial T}{\partial t}+\nabla \cdot(\mathbf{u} T)=\frac{\partial}{\partial z}\left(K_{T} \frac{\partial T}{\partial z}\right)+\frac{\partial Q_{s w}}{\partial z} \\
\frac{\partial S}{\partial t}+\nabla \cdot(\mathbf{u} S)=\frac{\partial}{\partial z}\left(K_{S} \frac{\partial S}{\partial z}\right),
\end{gathered}
$$

where $T$ is the temperature $\left({ }^{\circ} \mathrm{C}\right), S$ is the salinity, $K_{T}$ and $K_{S}$ are the vertical temperature and salinity diffusivity $\left(\mathrm{m}^{2} \mathrm{~s}^{-1}\right)$, and $Q_{s w}$ is the penetrative shortwave radiation flux $\left({ }^{\circ} \mathrm{C} \mathrm{m} \mathrm{s}{ }^{-1}\right)$. A nonlinear equation of state is used to relate total density, $\rho$, to $T, S$ and pressure (Feistel, 2008).

The model equations are discretized using a hexagonal-dominant unstructured horizontal grid (see Rayson et al., 2018) with fixed-height vertical (z-layer) coordinates. See Fringer et al. (2006) for an overview of the model discretization and numerical solution method.

\subsubsection{Model Parameterizations}

The surface, $z=\eta(x, y, t)$, and seabed, $z=-H(x, y)$, boundary conditions of the horizontal momentum Equations 13 and 14 are

$$
\left.v_{v} \frac{\partial \mathbf{u}}{\partial z}\right|_{z=\eta}=\frac{\vec{\tau}_{s}}{\rho_{0}},
$$



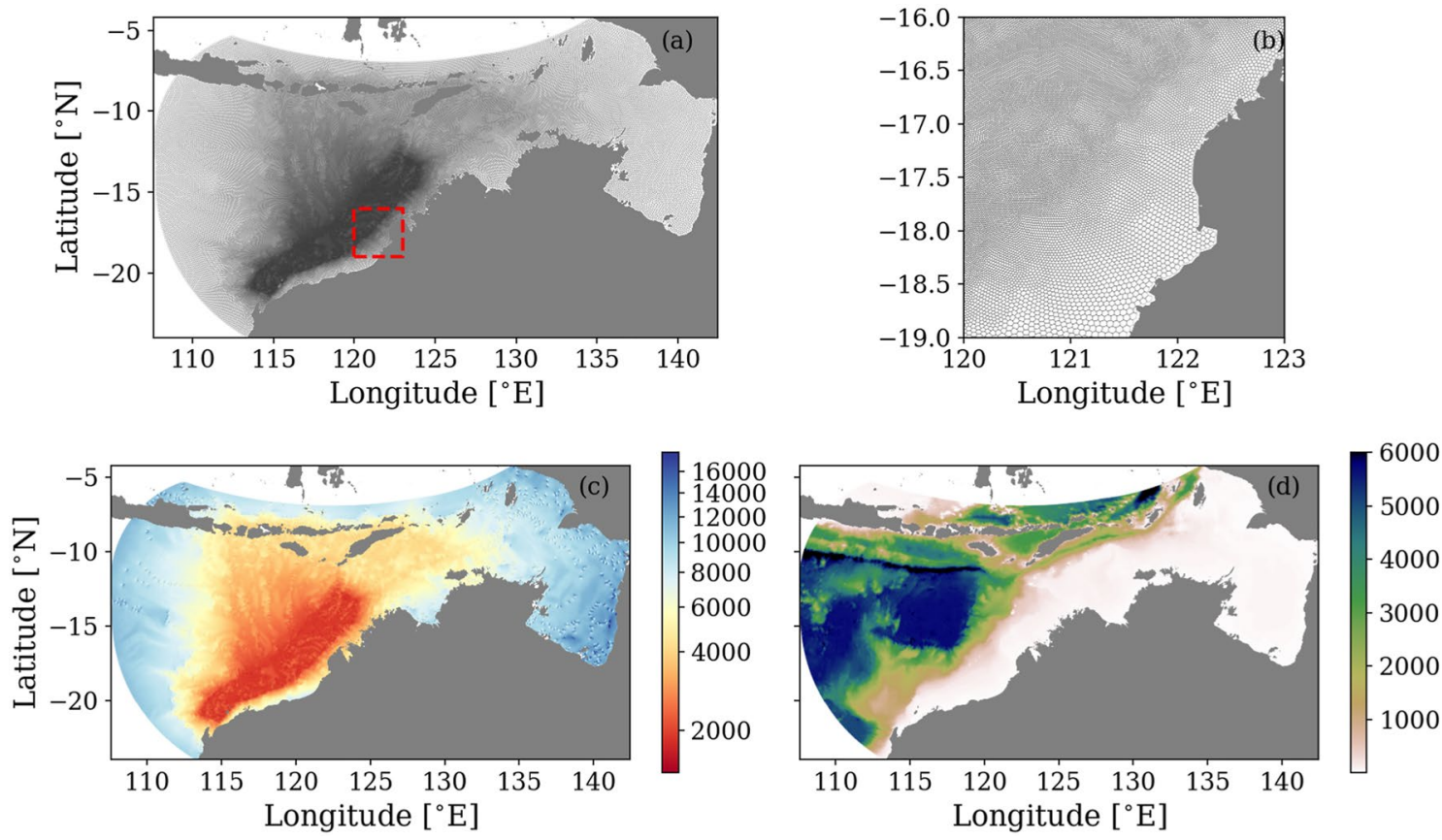

Figure 2. ( $\mathrm{a}$ and $\mathrm{b}$ ) Unstructured hexagonal-dominant SUNTANS mesh encompassing the Indo-Australian Basin, North West Shelf, Timor Sea, and Gulf of Carpentaria. (c) Horizontal grid resolution (m) noting that colors are on a nonlinear scale and (d) model bathymetry (m).

$$
\left.v_{v} \frac{\partial \mathbf{u}}{\partial z}\right|_{z=-H}=\frac{\vec{\tau}_{b}}{\rho_{0}},
$$

where $\vec{\tau}_{s}=\left(\tau_{x, s}, \tau_{y, s}\right)$ and $\vec{\tau}_{b}=\left(\tau_{x, b}, \tau_{y, b}\right)$ are the surface and seabed stress components, respectively. The surface stress is parameterized by

$$
\vec{\tau}_{s}=C_{d a} \rho_{a}\left|\mathbf{U}_{a}\right|\left(\mathbf{U}_{a}-\mathbf{u}_{\mid z=\eta}\right),
$$

where $\rho_{a}$ is the density of air $\left(1.2 \mathrm{~kg} \mathrm{~m}^{-3}\right), \mathbf{U}_{a}$ is the horizontal wind velocity vector, and $C_{d a}$ is the empirical surface drag coefficient. A quadratic drag formulation was also used to define the seabed stress

$$
\vec{\tau}_{b}=-\rho_{0} C_{d}\left|\mathbf{u}_{1 z=-H}\right| \mathbf{u}_{1 z=-H} .
$$

We used a quadratic bed drag coefficient of $C_{d}=0.002$. The surface drag coefficient was calculated using the COARE 3.0 algorithm (Fairall et al., 2003), which is wind speed dependent. See Rayson et al. (2015) for a thorough overview of the model surface heat, salt, and momentum boundary conditions. The horizontal eddy viscosity was constant $\left(v_{H}=1.0 \mathrm{~m}^{2} \mathrm{~s}^{-1}\right)$ and the vertical eddy viscosity and tracer diffusivities were computed with the Mellor and Yamada (1982) turbulence closure scheme.

\subsubsection{Grid}

The model domain encompassed the Australian North West Shelf, Timor Sea, and the southern Indonesian Archipelago because these are all known internal wave generation regions. The meridional span of the grid was $23^{\circ} \mathrm{S}$ to $5^{\circ} \mathrm{S}$ and the zonal span was $108^{\circ} \mathrm{E}$ (west of Western Australia) to E. The easternmost boundary was set to the shallow $(20 \mathrm{~m}$ ) Torres Strait off northern Queensland where there is limited volume exchange with the Coral Sea relative to the Indonesian Throughflow.

SUNTANS uses a finite-volume discretization of the governing equations and therefore employs an unstructured horizontal grid (Figure 2). We used a hexagonal-dominant grid that had the finest resolution (roughly $2 \mathrm{~km}$ ) over the North West Shelf and 4-km resolution in the Timor Sea and the major Indonesian passages of Timor, Ombai, and Lombok Straits (Figure 2b). The horizontal resolution telescoped out to about $10 \mathrm{~km}$ along all of the open boundaries, coinciding with the resolution of the ocean model used to force the model at the open boundaries (described below). The total number of horizontal grid cells was 225,368 . The unstructured grid can therefore efficiently span a large domain with the ability to focus reso- 
lution around a region of interest, namely the North West Shelf and the Indonesian-Australian Basin. Grid coordinates were projected in the World Mercator projection (EPSG 54004; https://epsg.io/54004) in order to perform metric distance calculations.

The vertical grid consisted of 80 layers with logarithmic stretching from the surface down to the deepest depth (capped at 6,000 m). The vertical resolution was roughly $7 \mathrm{~m}$ for the surface layer and each layer thickness increased on the last by a factor of 1.045, giving 20 layers in the upper $250 \mathrm{~m}$ and a vertical resolution of roughly $200 \mathrm{~m}$ in the abyssal ocean.

We compiled a new gridded bathymetry data set for the NWS and Indonesian Seas from several data sets, using a similar blending method to that described in Rayson et al. (2018). The input data sets were the Geoscience Australia (GA) 250-m grid from 2009, 50-m resolution multibeam data provided by GA and high-resolution multibeam data provided by Woodside Energy Ltd in selected regions over the NWS. The key difference between Rayson et al. (2018) is that here we used the General Bathymetric Chart of the Oceans (GEBCO) global 30 arc sec grid in the Indonesian Seas outside of the GA 250-m grid domain. Gridded bathymetry data were interpolated onto the unstructured grid cell centers (Figure 2a), and the maximum depth was capped at $6,000 \mathrm{~m}$.

\subsubsection{Model Boundary and Initial Conditions}

Background ocean state variables used for the SUNTANS initial and boundary conditions were sourced from the Mercator Ocean global reanalysis product, GLORYSv2. We used daily averaged temperature, salinity, and velocity variables and interpolated them in space and time onto our model grid points. The GLORYS reanalysis uses the NEMO ocean model with a 1/12th degree resolution global grid and 50 vertical $z$-levels. The model assimilates satellite sea surface height and temperature data, as well as in-situ data from ARGO profilers, moorings, and other sources. It is forced at the ocean surface by the ERA-interim atmospheric reanalysis product (described below).

Barotropic tidal velocity and free-surface boundary conditions were derived from the OTIS China and Indonesian Seas regional tide solution (Egbert \& Erofeeva, 2002). This regional tide solution has finer grid resolution $\left(1 / 30^{\circ}\right)$ than the global solution $\left(1 / 4^{\circ}\right)$ and is therefore able to resolve the Indonesian Archipelago topography in greater detail to provide better tidal predictions (Stammer et al., 2014). Time-varying velocity fluxes and free-surface elevations were reconstructed from eight tidal constituents, namely $M_{2}, S_{2}, N_{2}, K_{2}$, $K_{1}, O_{1}, P_{1}, Q_{1}$, at the SUNTANS open boundary edges. Tidal fluxes were added to the low-frequency (daily average) open boundary velocities interpolated from the GLORYS reanalysis.

Atmospheric data from the European Centre for Medium Range Weather Forecast's (ECMWF) ERA-Interim climate reanalysis product was used to drive the exchange of momentum and heat between the atmosphere and the model ocean. ERA-Interim is a global, data-assimilating atmospheric hindcast model run on a roughly 100-km grid with output data stored at six-hourly time steps (Dee et al., 2011). Air-sea fluxes are parameterized in SUNTANS using the COARE3.0 algorithm using east-wind and north-wind velocity referenced to $10 \mathrm{~m}$ above the surface, air temperature, pressure, and relative humidity (Fairall et al., 2003). Net longwave and shortwave radiation components are calculated internally within the model using cloud cover from ERA-Interim and model latitude and time to compute the solar input (see Rayson et al. (2015) for a description of the numerical implementation of the heat flux module in SUNTANS).

\subsection{Validation of Low-Frequency Temperature Stratification}

We first tested the performance of the ocean model to reproduce the low-frequency evolution of the temperature stratification on the shelf by comparison with through-water-column temperature at the four different shelf locations. Model variables were saved at the observation sites with the same temporal sampling interval $(60 \mathrm{~s})$. We then linearly interpolated model data onto the observation depths. Temperature bias and root mean square error (RMSE) were computed for three different months to evaluate the model performance at capturing the seasonal surface layer and thermocline variations over the region. At the ITFTIS mooring, the model did well at replicating the surface heating and cooling from September 2013 to June 2014, as well as the mixed layer deepening in June (Figure 3). Bias in the upper $100 \mathrm{~m}$ was generally close to zero and the RMSE was $<0.5^{\circ} \mathrm{C}$. Model performance was generally worse in the thermocline between $100-\mathrm{m}$ and $300-\mathrm{m}$ 

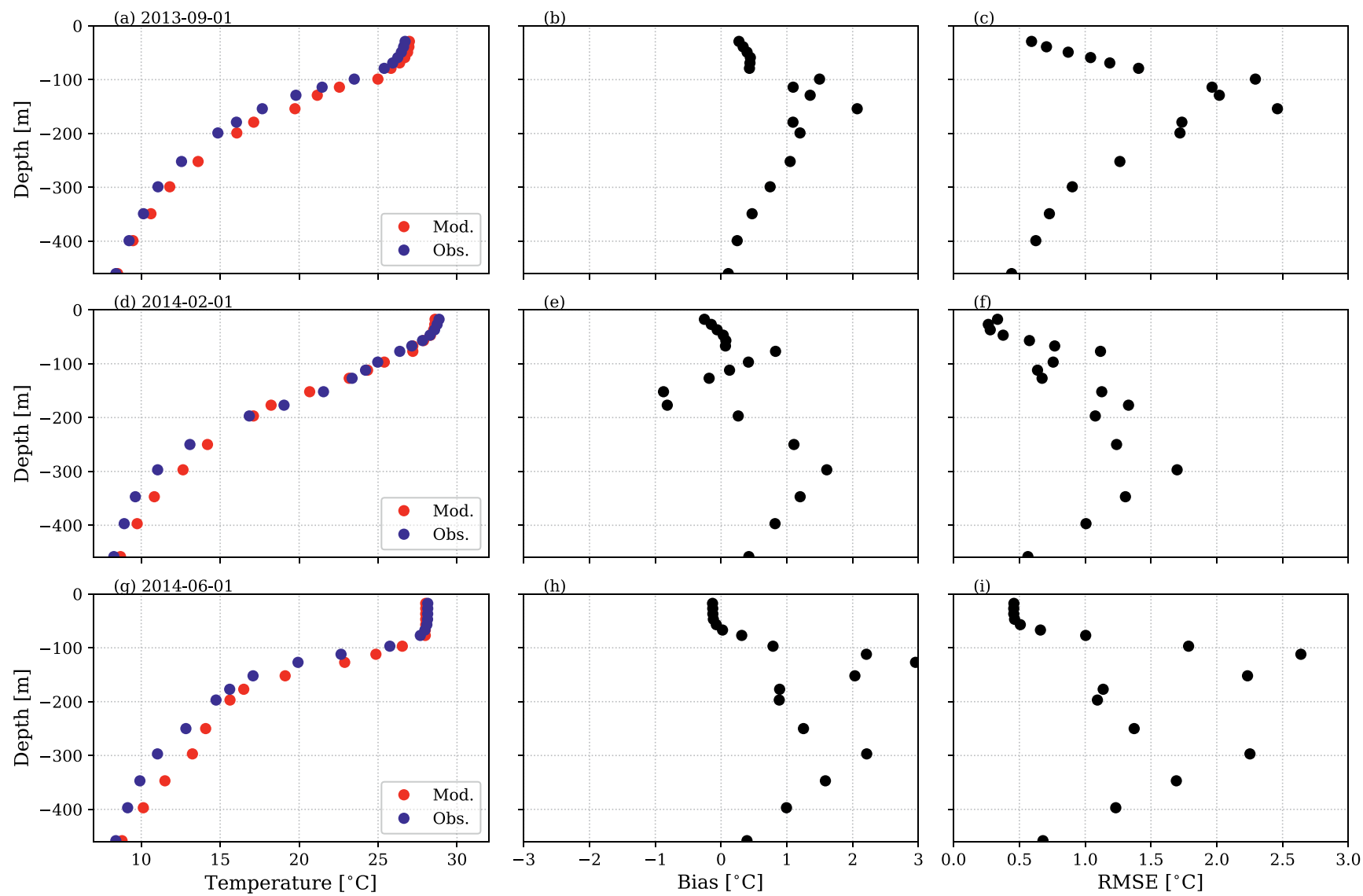

Figure 3. Quantitative SUNTANS model evaluation metrics against in-situ temperature observations from the ITFTIS mooring of (left column) monthly averaged temperature, (middle column) temperature bias, and (right column) temperature root mean square error. Each row corresponds with monthly averages for September 2013, February 2014, and June 2014.

deep. At the ITFTIS mooring, the model exhibited a $1-3{ }^{\circ} \mathrm{C}$ warm bias that was most pronounced during June 2014. The RMSE was also higher in the thermocline where there were large high-frequency temperature variations due to internal tides. Higher RMSE at these depths were therefore reflective of both mean and internal tide-induced model-data mismatch. Note that the higher RMSE in the thermocline was also because the model used the hydrostatic approximation and had insufficient horizontal resolution to capture high-frequency, nonlinear internal waves that were present in the observations.

The temperature bias was generally less at the PIL200 and KIM200 shelf sites $\left( \pm 1^{\circ} \mathrm{C}\right)$, while at the KIM400 site the model exhibited a $1-3^{\circ} \mathrm{C}$ cool bias in the thermocline between 100 and 300 (not shown). The analysis at all moorings indicated, however, that there was no systematic temperature bias (i.e., too hot or too cold) throughout the whole model domain; any biases were specific to each individual mooring. Poorer validation statistics in the thermocline were due to a $20-50 \mathrm{~m}$ offset in the thermocline depth and admittedly, there is room for improvement in this aspect. Accurately capturing the thermocline structure and strength, however, is an on-going major challenge for all ocean/climate models (e.g., Castaño-Tierno et al., 2018). Overall, the model performed well at capturing the seasonal evolution of near-surface temperature and mixed layer development at each site. It also captured seasonal fluctuations in thermocline strength and width-the main ocean properties likely to temporally modulate internal tides on a regional scale. 

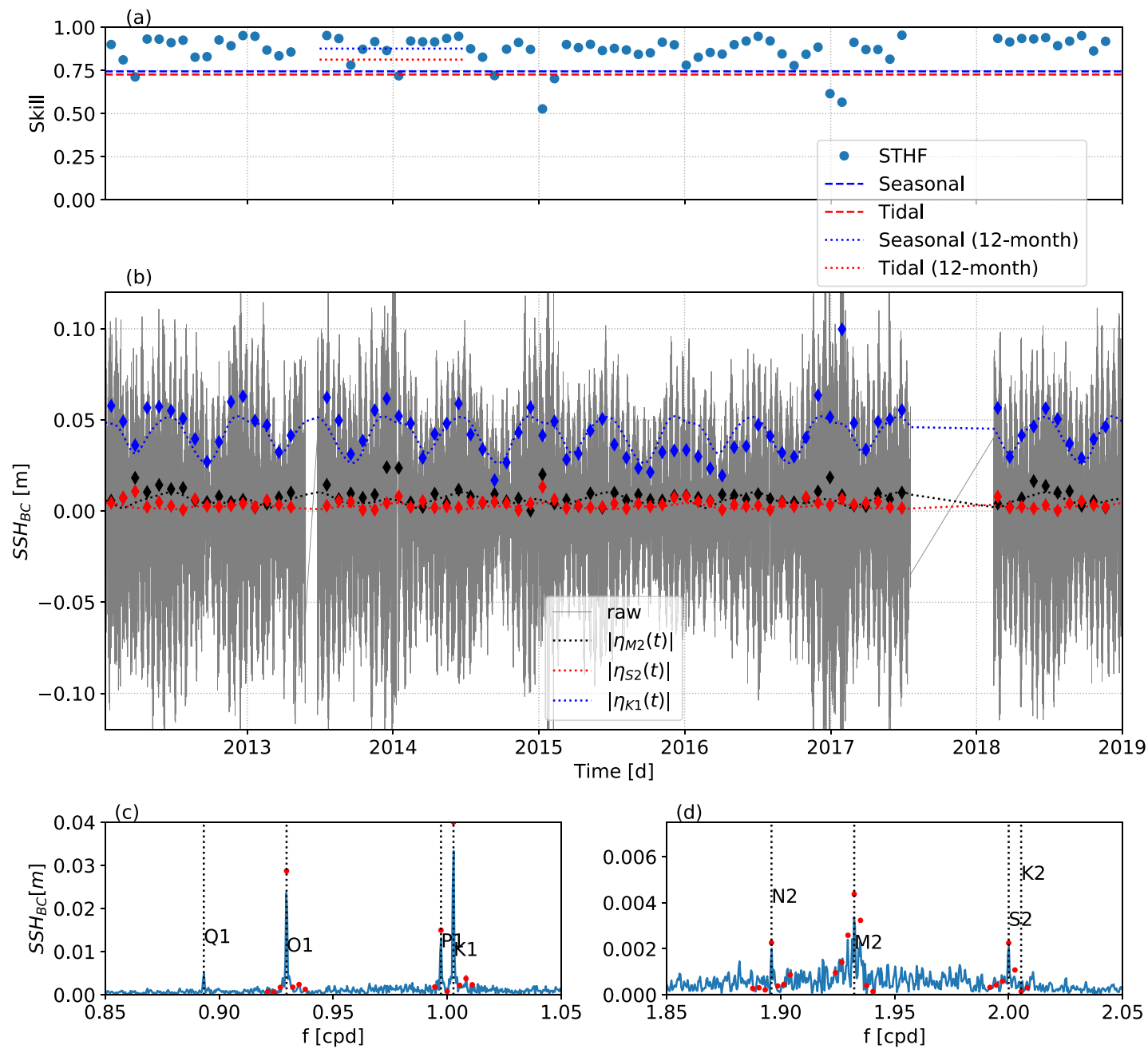

Figure 4. (a) Harmonic model skill scores and (b) baroclinic sea surface height (m) at the Timor Sea (ITFTIS) mooring. Diamonds in (b) indicate amplitudes of the three major tidal constituents from 30-day STHF while the dashed lines indicate the seasonal harmonic model fit amplitude (Equation 5). (c and d) are the discrete Fourier transform amplitude for the diurnal and semidiurnal bands, respectively (note the change in vertical scale). Red dots in (c and d) indicate the least-squares fit amplitude of the tidal bands plus the annual harmonics. Frequencies of the eight major tidal constituents are indicated by the vertical dotted lines.

\section{Observations of Seasonal Internal Tides}

We performed four separate analyses of $\mathrm{SSH}_{B C}$ inferred from the multiyear in-situ observation records to investigate the seasonal modulation of the internal tides. These analyses were: (a) short-time harmonic fits (denoted as STHF) to 30-day windows of data with 50\% overlap using five astronomical tidal constituents $\left(M_{2}, S_{2}, N_{2}, K_{1}\right.$, and $\left.O_{1}\right)$; (b) discrete Fourier transform of the whole record where data gaps were filled using linear interpolation; (c) least-squares fit with seasonal harmonic model using the same five astronomical constituents and three annual harmonics; and (d) least-squares harmonic fit using six major astronomical constituents $\left(M_{2}, S_{2}, N_{2}, K_{1}, O_{1}, P_{1}\right)$. The record length used in the above analyses for each mooring are listed in Table 1 . We refer to the latter two harmonic fits as the seasonal and tidal harmonic models. Note that we explicitly included the $P_{1}$ constituent in the tidal model while it is implicitly included in the seasonal model.

We use the Murphy Skill score as a harmonic model performance evaluation metric

$$
\text { skill }=1-\frac{\sum\left(X_{m o d}-X_{o b s}\right)^{2}}{\sum\left(X_{o b s}-\mu_{o b s}\right)^{2}},
$$


where $X_{o b s}$ and $X_{m o d}$ are the observed and model quantities, respectively, and is the mean observed quantity. Here, a modeled quantity means predicted using harmonics. Skill score is equivalent to the fraction of variance explained by the model e.g., skill $=0.8$ corresponds to $80 \%$ of the signal variance being explained by the model.

\subsection{Analysis of Inferred $S S H_{B C}$ From In-Situ Observations}

The ITFTIS (Timor Sea) mooring experienced the largest $S S H_{B C}$ of the four sites examined, with values exceeding $12 \mathrm{~cm}$ (Figure 4). The STHF revealed that the $K_{1}$ diurnal constituent was largest and fluctuated on a seasonal scale of two cycles per year (blue diamonds in Figure $4 \mathrm{~b}$ ). The reconstructed $K_{1}$ time series using Equation 5 and the seasonal harmonic model sidelines (i.e., $\left|\eta_{K 1}\right|$ ) also revealed semiannual oscillations (dashed blue line). Both the discrete Fourier transform amplitude and the seasonal harmonic model confirmed a peak at $\omega=\omega_{K 1}-2 \omega_{A}$, corresponding with the $P_{1}$ astronomical constituent (Figure $4 \mathrm{c}$ ). Note that Equation 6 shows the relationship between the annual harmonics and the spectral content of the signal. Annual harmonics have an equivalent frequency offset by $\pm n \omega_{A}$ from each tidal frequency and, since we use $N=3$ harmonics, there are six additional discrete spectral peaks around each tidal constituent (Figures $4 \mathrm{c}$ and $4 \mathrm{~d})$. The $M_{2} S S H_{B C}$ component was an order of magnitude smaller than $K_{1}$, however, the annual frequency amplitudes $\left(M_{2} \pm \omega_{A}\right)$ were significant, at roughly $50 \%$ of the $M_{2}$ amplitude (Figure $4 \mathrm{~d}$ ). This resulted in seasonal variations of the $M_{2}$ harmonic amplitude, $\eta_{M 2}$ in Figure 4b. Note that Kelly et al. (2014) reported seasonal variations in the $M_{2}$ baroclinic current amplitude at this site, not $S S H_{B C}$, attributing it to seasonal variations in stratification.

Interannual variations in the $K_{1}$ amplitude were identified by the mismatch between the STHF and the seasonal $K_{1}$ amplitude modulation in Figure 4b. The interannual variations were present during late 2013 and early 2016, for example, and suggest that the $K_{1}$ internal tide amplitude variations were caused by background ocean processes, and not directly due to tidal forcing. Likewise, there were periods when the $M_{2}$ amplitude from the STHF deviated substantially from the seasonal model. Last, note that the skill of the STHF for 30-day windows varied between 0.50 and 0.95 at this site indicating there were short periods that were less predictable using just fixed-amplitude tidal harmonics (Figure 4a). The skill of the multiyear tidal and seasonal harmonic model fits is discussed in the next section.

Moorings KIM200 and KIM400 were located on the Kimberley Shelf in 200-m and 400-m water depths, respectively. The moorings were separated by $38 \mathrm{~km}$ and we analyzed 2-year of data collected between July 2012 and August 2014. Analysis of the $S S H_{B C}$ signal revealed that the $M_{2}$ amplitude was dominant $(2.5 \mathrm{~cm})$ at KIM200 and there were peaks at annual and triannual cycles (Figure 5). Phasing of the seasonal variations resulted in a peak $M_{2}$ amplitude around January and April of each year (Figure $5 \mathrm{~b}$ ). The other four tidal frequencies were all $<75 \%$ of the magnitude of $M_{2}$, except for $K_{1}$, which had triannual peaks in August, January, and May that were roughly $50 \%$ of the $M_{2}$ amplitude. Note that during October and November of both 2012 and 2013, the $M_{2}$ amplitude decreased and was similar in magnitude to $S_{2}$. The STHF skill varied between 0.5 and 0.8 at this site, with the peaks in skill coinciding with peaks in $\left|\eta_{M 2}\right|$ (October and April, Figure 5a). The interannual variations were small for the 2-year record and the seasonal model captured the amplitude variations calculated using the STHF (dashed lines and diamonds in Figure 5b). The $S_{2}$ harmonic amplitude was next greatest $(1 \mathrm{~cm})$ followed by $K_{1}$ and $O_{1}$ harmonics $(0.8$ and $0.6 \mathrm{~cm}$, respectively). All constituents had seasonal side line amplitudes that were at least $10 \%$ of the main astronomical forcing frequency amplitude.

In contrast to the KIM200 site, the KIM400 $S S H_{B C}$ had a small $M_{2}$ amplitude $(0.2 \mathrm{~cm})$ and a dominant $K_{1}$ constituent (Figure 6). The $M_{2}$ amplitude at KIM400 was about 25\% of the magnitude of KIM200 (Figure 6b). The skill of the STHF was also lower than KIM200, on average, with values between 0.25 and 0.70. The diurnal $K_{1}$ amplitude was similar in amplitude to the KIM200 site, although the magnitude of the seasonal harmonics differed, e.g., the $P_{1}\left(\omega_{K 1}-2 \omega_{A}\right)$ amplitude was roughly $50 \%$ smaller at KIM400. There was also a peak of similar magnitude at frequency $K_{1}+\omega_{A}$.

The PIL200 mooring, located on the southern region of the NWS, had dominant $M_{2}$ and $K_{1}$ amplitudes of 1.5 and $1.3 \mathrm{~cm}$, respectively (Figure 7). Annual harmonics of these dominant constituents, and the other frequencies, were significant at this site. For example, the $M_{2}-3 \omega_{A}$ frequency had an amplitude of $0.7 \mathrm{~cm}$ 

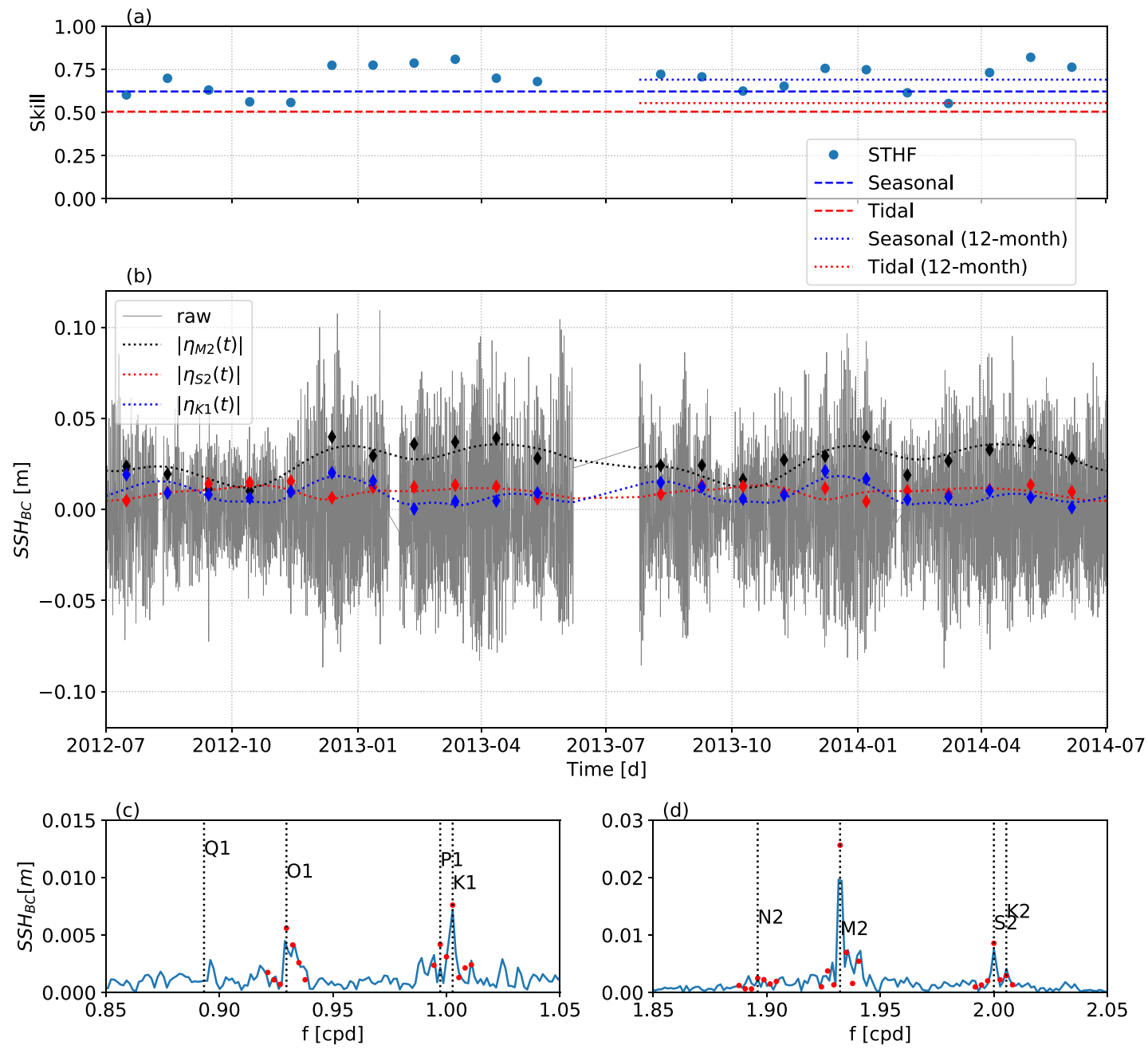

Figure 5. Similar to Figure 4 but for the KIM200 mooring. Note the different time scales in (a) and the different vertical scales in (c and d).

(roughly $50 \%$ of the $M_{2}$ amplitude) while $K_{1}-\omega_{A}$ was roughly $40 \%$ of the $K_{1}$ amplitude. It can be seen from the difference between the STHF and the seasonal harmonic $M_{2}$ amplitude (black diamonds and black dashed line, respectively, in Figure $7 \mathrm{~b}$ ) that the seasonal model did not capture all of the month-to-month variations in amplitude. It did, however, perform $50 \%$ better than the model with six major fixed-amplitude astronomical harmonics. The seasonal sidelines explained the annual increase in $\left|\eta_{M 2}\right|$ to $3 \mathrm{~cm}$ around July each year, e.g.

The seasonal oscillations of the internal tide harmonics exhibited the most complex behavior at the PIL200 site (Figure $7 \mathrm{~b}$ ). The $M_{2}$ and $K_{1}$ bands were dominant although their relative importance varied significantly throughout the year. The $M_{2}$ band had triannual peaks in July, October, and March, while the $K_{1}$ band had a semiannual cycle with peak amplitude in December and July due to the $P_{1}$ sideline. This resulted in $K_{1}$ dominating at PIL200 between October and February, while $M_{2}$ was dominant for the other periods of the year. The exception being during September, when $M_{2}, N_{2}$, and $K_{1}$ were of equal magnitude.

\subsection{Seasonal Harmonic Model Evaluation}

We quantified the amount of internal tide $S S H_{B C}$ signal variance captured with both harmonic models (i.e., with and without seasonal harmonics) by comparing the skill at the four multiyear observation locations (ITFTIS, KIM200, KIM400, and PIL200). We first evaluated the skill for the multiyear record at each moor- 

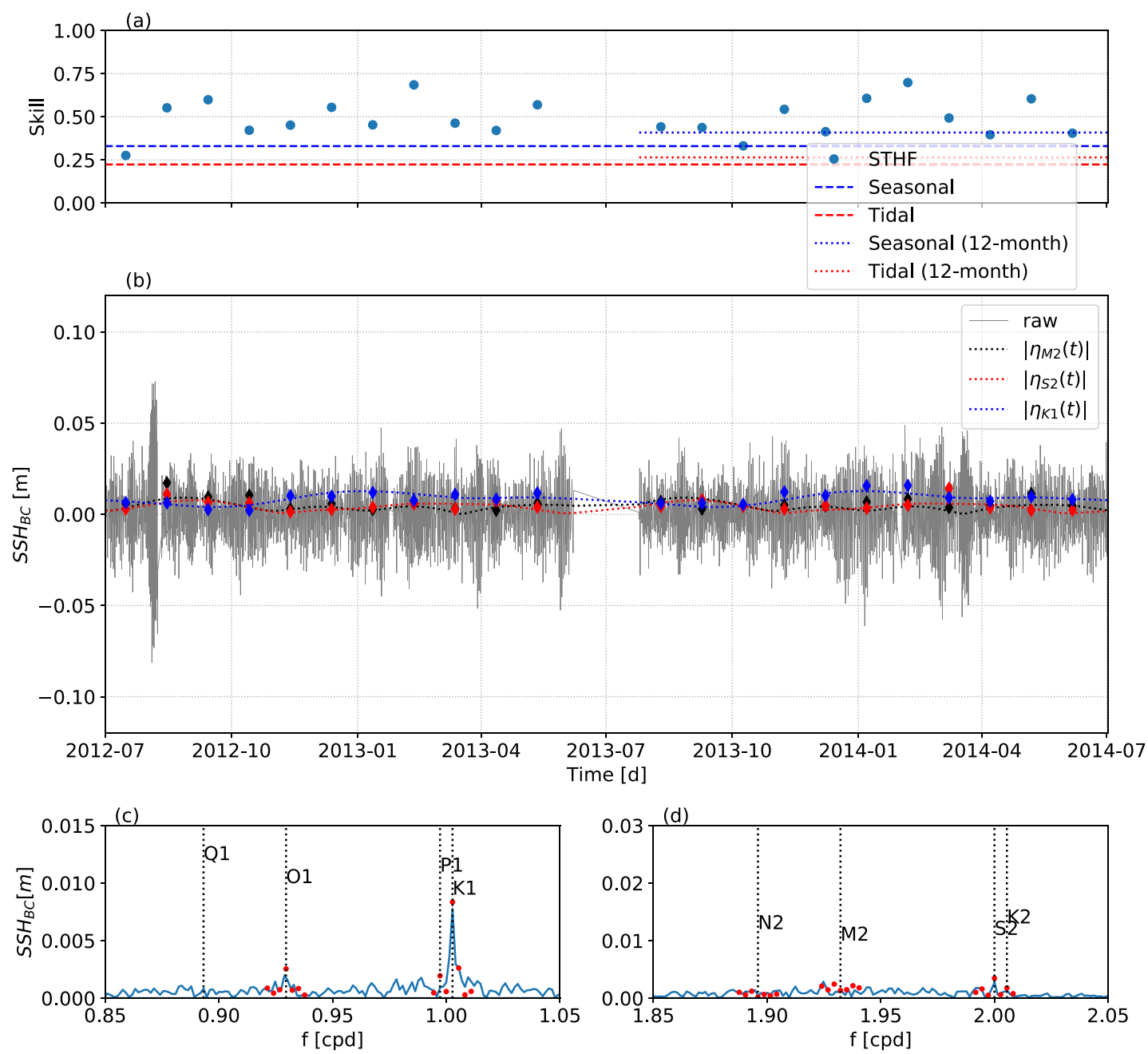

Figure 6. Similar to Figure 4 but for the KIM400 mooring. Note the different time scales in (a) and the different vertical scales in (c and d).

ing, and then for a 12-month period only (July 2013 to August 2014). The purpose of fitting to a 12-month period was to make a robust comparison of skill between the different sites and to assess the importance of interannual variability. All skill scores here are computed using the same record length that was used to compute the harmonic amplitudes.

The seasonal harmonic model had a higher skill (i.e., explained more variance) than the tidal model at all four sites (Table 2). There are differences between sites, and the skill scores are presented in panel (a) of Figures $4-7$. At ITFTIS, the seasonal model had a skill score of 0.74 compared with 0.73 for the tidal model when fit to 7 years of data, and corresponding skills of 0.90 and 0.81 for a 12 -month period. The differences suggest that interannual variations in the seasonal harmonics are likely important at this site and the seasonal model was partially capturing the semiannual oscillation in $S S H_{B C}$ (discussed previously). The ITFTIS was the best performing (most predictable), site followed by KIM200 (skill = 0.62), PIL200 (skill = 0.38), and KIM400 (0.33). The improvement in skill between the two models varied between sites: the increase was $0.01,0.11,0.11$, and 0.13 for sites ITFTIS, KIM200, KIM400, and PIL200, respectively, indicating the relative importance of the seasonal sidelines at different locations. At PIL200 and KIM400, this amounted to a roughly 50\% improvement in the total amount of variance captured when including the seasonal sidelines.

We used the skill of the 12-month fit to both harmonic models to make a fairer comparison between the mooring sites (Table 2). The skill of both harmonic models improved for the 12-month period, which is 

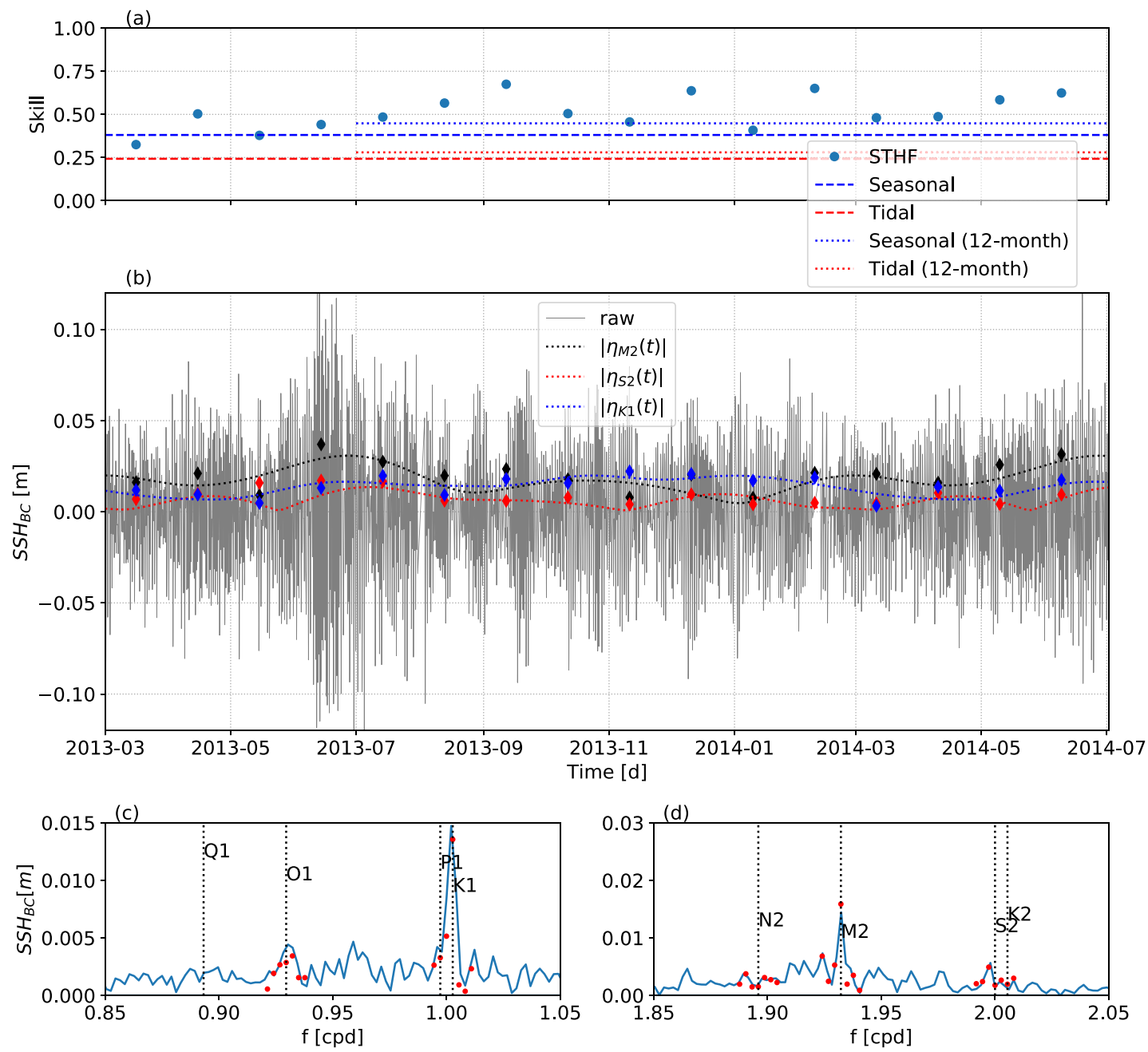

Figure 7. Similar to Figure 4 but for the PIL200 mooring. Note the different time scales in (a) and the different vertical scales in (c) and (d).

expected because the seasonal amplitudes were not averaged over multiple years. Furthermore, the skill difference between the seasonal model and the tidal model was greater for the 12-month fit, and this indicates that the seasonal model performance degraded with increased record length. The skill difference for the 12-month records was $0.09,0.13,0.14$, and 0.17 for sites ITFTIS, KIM200, KIM400, and PIL200, respectively. Again, the greatest increases in skill with the inclusion of the seasonal harmonics were at KIM400 and PIL200.

\section{Table 2}

Performance Metrics of the Tidal (Equation 1) and Seasonal (Equation 6) Models at Predicting the Sea Surface Height Perturbation at Each of the Mooring Locations for the Record Period and for the 12-Month Period Spanning July 2013 to July 2014

\begin{tabular}{lcccccc}
\hline Site & Start date & End date & Seasonal & Tidal & Seasonal (12-month) & Tidal (12-month) \\
\hline ITFTIS & January 01, 2012 & January 01, 2019 & 0.74 & 0.73 & 0.90 & 0.81 \\
KIM200 & August 01, 2012 & August 01, 2014 & 0.62 & 0.51 & 0.69 & 0.56 \\
KIM400 & August 01, 2012 & August 01, 2014 & 0.33 & 0.22 & 0.41 & 0.27 \\
PIL200 & March 01, 2013 & August 01, 2014 & 0.38 & 0.24 & 0.45 & 0.28 \\
\hline
\end{tabular}




\subsection{Summary of Observations}

The key insights gained from the empirical harmonic analysis of the in-situ mooring data at four locations along the shelf were:

1. Internal tide predictability using either major tidal astronomical amplitude harmonics, or time-varying seasonal harmonics was best explained at the ITFTIS and KIM200 sites

2. The STHF skill varied in time at each site indicating internal wave variability outside of the major astronomical (forcing) frequency bands

3. Seasonal harmonics explained up to $50 \%$ more variance at the KIM400 and PIL200 moorings, although these sites were the least predictable, overall

4. The seasonal harmonic model performed better when applied to a 12-month period than the multiyear record, suggesting interannual variability is important (but currently unaccounted for)

5. KIM200 was the only one of the four sites with a dominant $M_{2}$ internal tide for the whole observation record, despite $M_{2}$ barotropic tides being dominant on the shelf

6. KIM400 had roughly 50\% smaller amplitude internal tides than KIM200 despite being located only $38 \mathrm{~km}$ away

7. PIL200 had poor predictability and complicated seasonal variability, such as the dominance of different harmonics throughout the year

\section{Model Interpretation of the Seasonal Internal Tide Variability}

We now use the numerical model results to help interpret the geographic and temporal variability in the observed seasonal internal tide harmonics. The model was forced with eight discrete tidal frequencies so any temporal variability in the $M_{2}$ harmonic, for example, can be attributed to an unforced (nonlinear) response.

\subsection{Statistical Overview of the Seasonal Harmonics}

We analyzed the internal tide variability from the 12-month regional ocean model solution by first calculating $S S H_{B C}$ from water density using the steric height definition (Equation 12). The regional map of $S S H_{B C}$ variance (Figure 9a) had qualitatively similar spatial features as the $M_{2}$ amplitude estimates from satellite altimetry (e.g., Figure 1). Regions of large variance (e.g., on the NWS and near the Indonesian straits) correspond with significant internal tide generation zones, whereas banding patterns of low and high variance indicate constructive and destructive interference patterns caused by waves propagating in multiple directions; these are standing wave antinodes and nodes, respectively.

The ability of the seasonal harmonic model to capture the internal tides was quantified using both the variance of the residual (Figure 9b) (i.e., the mean squared error) and the skill (SHVF in Equation 8, Figure 9c) (note that Figure $9 \mathrm{c}$ is equal to one minus Figure $9 \mathrm{~b}$ divided by Figure $9 \mathrm{a}$ ). The residual variance was largest in an arc decreasing southward of Indonesia and in isolated patches on the NWS, such as near the Rowley Shoals. Many of these regions corresponded to regions of large total signal variance. The skill score (SHVF) allows for a relative comparison between sites with different internal tide amplitudes because it normalizes the mean squared error by the variance of the signal. The seasonal model captured $50-100 \%$ of the variance over the vast majority of the study region. The exception being the shelf regions $<100-\mathrm{m}$ deep where the water depth is too shallow to support year-round internal wave propagation, and the region south of the Indonesian islands. The drivers of the variability in this region will be explored in Section 6 . The difference between SHVF and THVF highlights regions where seasonal harmonics are relatively more important (Figure 10). The importance of seasonal harmonics was highly spatially variable with decorrelation length scales of about one internal wavelength. The seasonal model helped explain more variance in nodal regions (described below) such as the KIM400 mooring site. On the shelf and slope region between 200-m and 500$\mathrm{m}$ water depth, where all of the mooring sites examined here were located, the SHVF parameter explained 10-30\% more of the $\mathrm{SSH}_{B C}$ variance in the model (see also Table 4). PIL200 also straddled a standing internal wave node. On the shelf in water depths 100-200 m, the seasonal model explained up to 50\% more of the 

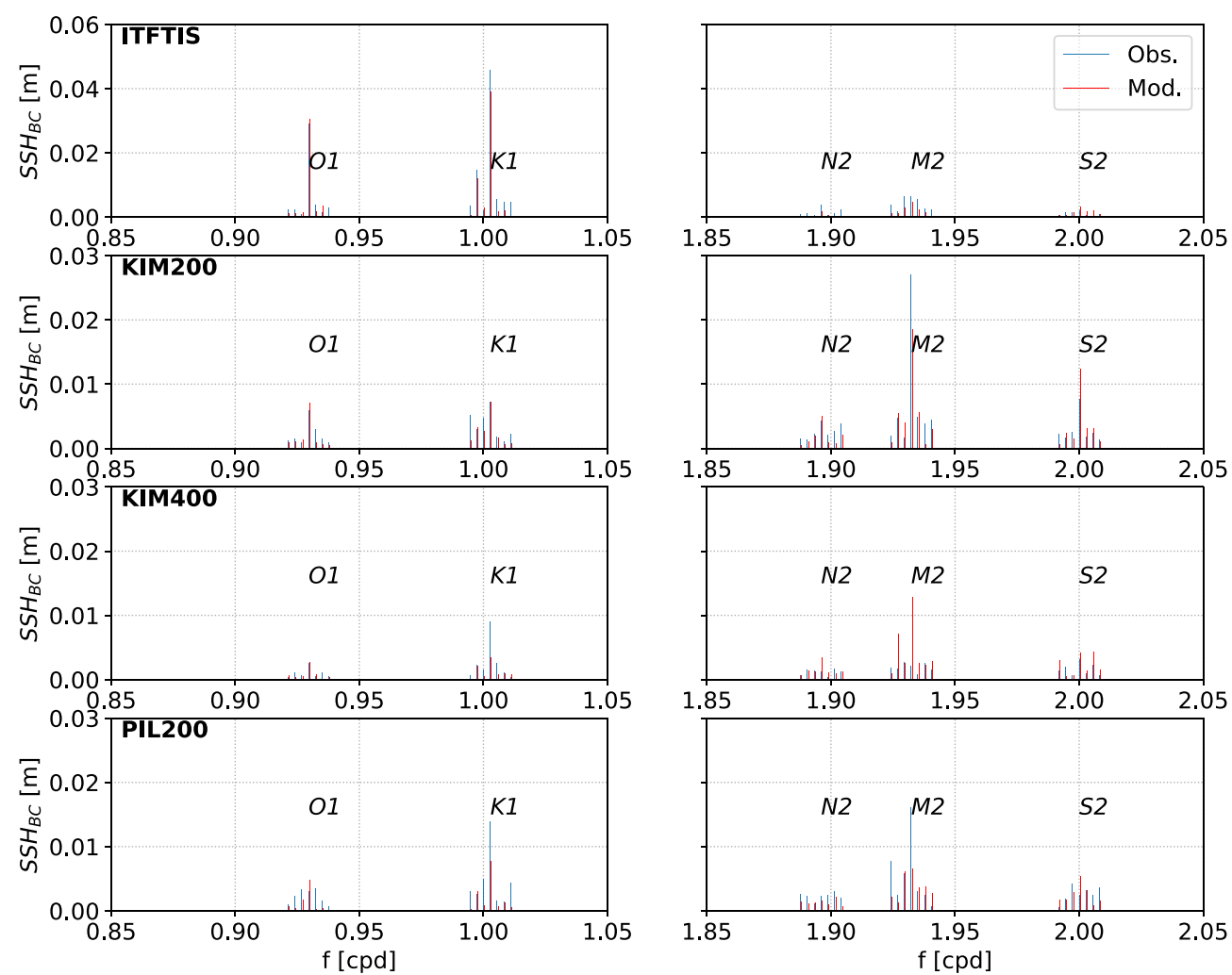

Figure 8. $S S H_{B C}$ amplitudes of the discrete harmonics in the seasonal model from the observation data (blue) and model result (red). Each row represents each site, and each column contains the diurnal and semidiurnal harmonics. Note the different vertical scales for the ITFTIS site.

signal variance. This was significant as the tide-only harmonic fit explained close to zero percent of signal variance in these depths (not shown).

These results suggest that the internal tides on the shelf were more sensitive to seasonal stratification changes than in the deep ocean basin, as would intuitively be expected in relatively shallow regions where the stratification can vary strongly over a year and even disappear completely on occasion. The region of the Indo-Australian Basin spanning 110-115 E and 15-10 S was another region where the seasonal effects were important. In this region, the model residual variance was relatively large (3-4 $\mathrm{cm}^{2}$, Figure 9b), SHVF was 50-80\% (Figure 9c), and the contribution of seasonal harmonics described $>50 \%$ of the $S_{S C}$ variance (Figure 10). We explore the potential physical drivers of the seasonal modulation of the internal tide amplitude in this particular region in Section 6 by relating the internal tide response to the large-scale circulation.

Another metric for identifying the importance of the seasonal terms, the seasonal variance fraction $\left(S V F_{m}\right)$, generally peaked in standing wave node regions for both the $M_{2}$ and $K_{1}$ bands (Figure 11). The seasonal variance fraction was large in water depths $<200 \mathrm{~m}$ where seasonal variations in the surface mixed layer depth can eliminate stratification, and hence internal waves, resulting in a mean amplitude close to zero. The less predictable mooring sites (in terms of the seasonal skill score (SHVF) in Table 3) also exhibited greater $S V F_{M 2}$ e.g., it was 59\% at PIL200 and 40\% at KIM400 (Table 4).

The variance fraction of the harmonic signals in the $M_{2}$ and $K_{1}$ bands $\left(V F_{m}\right)$ closely resembled the mean harmonic amplitude with $M_{2}$ dominance (60-90\% of variance) on the NWS and throughout the Indo-Australian Basin (Figure 12a). Conversely, $V F_{K 1}$ was dominant in the Timor Sea. There were, however, isolated patches where this general picture was violated. A notable example was the prevalence of the $K_{1}$ band around PIL200 where $V F_{K 1}$ was roughly $50 \%\left(V F_{M 2}\right.$ and $V F_{K 1}$ were $43 \%$ and $23 \%$, respectively; Table 4$)$. Likewise, there were regions of the Timor Sea, away from ITFTIS, where $V F_{M 2}>50 \%$, whereas $V F_{M 2}$ was only 

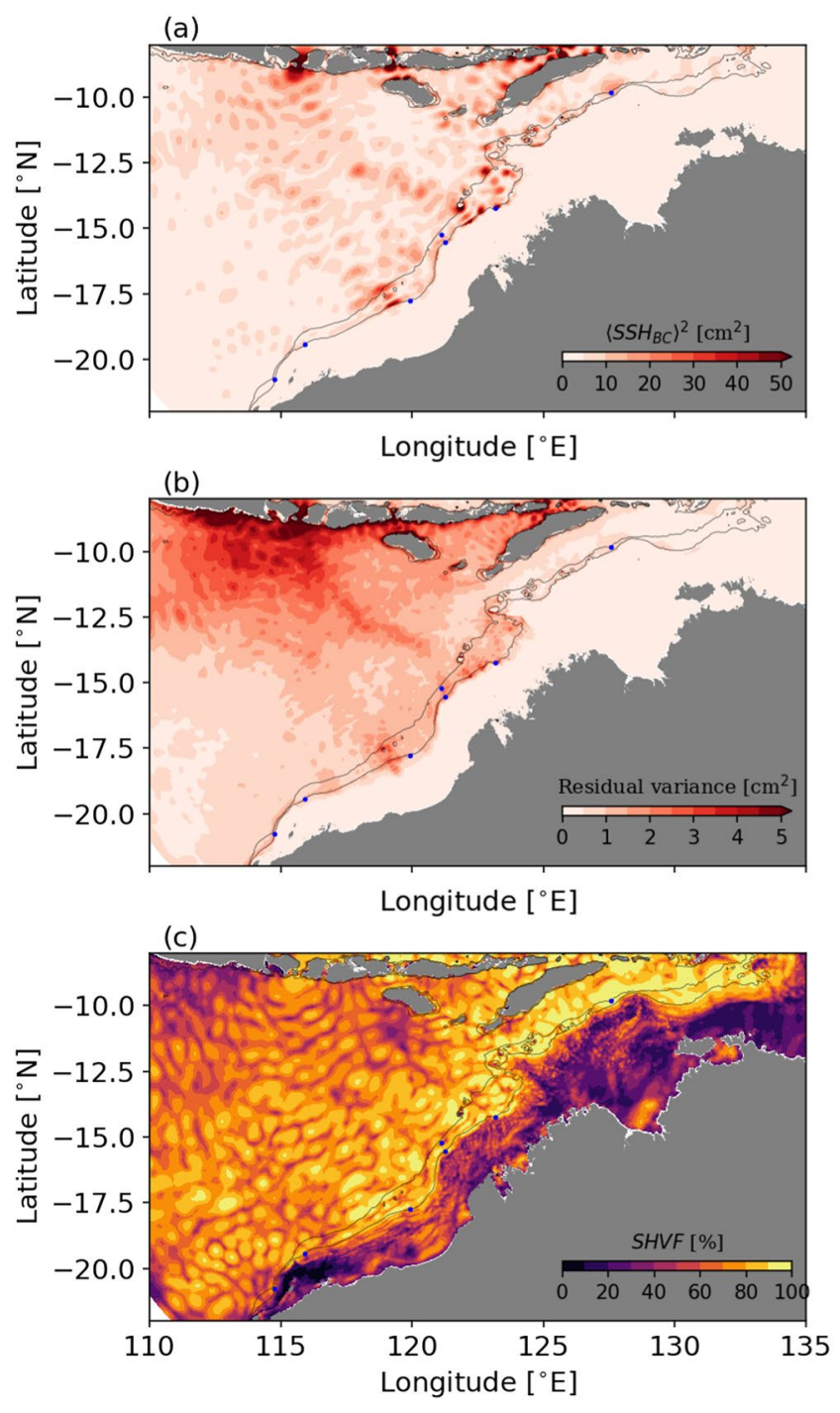

Figure 9. (a) Total variance of $S S H_{B C}$ signal from 12-month of hourly model snapshots, (b) variance of the residual between the seasonal harmonic model and the raw quantity, and (c) percentage of variance of the SUNTANS $S S H_{B C}$ explained by the seasonal harmonic model (SHVF, Equation 8).
$2 \%$ at ITFTIS. These isolated patches emphasize why individual moorings may not be representative of the broader regional variability of internal tide-induced sea level fluctuations.

\subsection{SUNTANS Internal Tide Evaluation}

We validated the internal tides generated in the 12-month SUNTANS solution, by first calculating the seasonal harmonic amplitude parameters of $\mathrm{SSH}_{B C}$ at each horizontal grid point using least-squares, and then directly compared them with the amplitudes derived from observations for the concurrent period (Figure 8). This representation shows the relative amplitude of the seasonal harmonics compared to the main astronomical frequencies. The ITFTIS site, e.g., was dominated by $K_{1}, O_{1}$, and $P_{1}\left(K_{1}-2 \omega_{A}\right)$ frequencies, and the SUNTANS-derived harmonics replicated this observation. The relative contributions of the seasonal harmonics at the other sites were obvious in both the observation-derived and model-derived harmonics. At KIM200, the $M_{2}$ signal was the major frequency yet the seasonal sidelines were $20-30 \%$ of this amplitude. The model-derived $M_{2}$ amplitude was about $0.7 \mathrm{~cm}(40 \%)$ smaller than the observed amplitude at KIM200, whereas it was $0.7 \mathrm{~cm}$ larger at KIM400. Conversely, the model-derived $K_{1}$ amplitude was 50\% smaller at KIM400. We suggest that these model-observation mismatches in amplitude are likely due to subtle differences in the spatial locations of constructive and destructive wave interference zones (i.e., standing wave nodes and antinodes). Details of this phenomenon are presented below using spatial fields of the harmonic amplitudes.

We also assessed the predictive skill of the spatial harmonics derived from the 3D primitive equation ocean model by directly comparing with observations (at ITFTIS, KIM200, KIM400, and PIL200) collected during the run period of the model, and also with observations collected outside of this period (NWSBAR, NWSROW, and NWSBRW moorings). Note that here we are comparing results with the band-passed filtered observation data, not the harmonically reconstructed observation data. The purpose here is to assess whether the seasonal harmonic climatology is useful at new locations and for different time periods. At most sites, the skill score was greater than zero, indicating some predictive capability of the SUNTANS-derived harmonic model (Table 3). The best predictions were (in descending order) at NWSBRW, NWSROW, and ITFTIS sites with skill scores of $0.67,0.45$, and 0.47 , respectively. These were also generally regions of larger total internal tide amplitude (as shown below). The poorest predictions were at the KIM400, PIL200, and NWSBAR sites with skill scores of $-0.60,0.02$, and 0.12, respectively. These results indicate poorer predictive skill of a seasonal harmonic model (and SUNTANS) along the southern (Pilbara) section of the NWS.

Generally, the SUNTANS-derived internal tides were weaker in magnitude when compared to the observed major constituents at all sites. The only exception being at KIM400 (Figure 8). Given the strength of the barotropic tidal forcing was skillfully captured by the model, the weaker modeled internal tides were likely due to a combination of effects including: biases in the mean thermocline properties; errors in bathymetry; discretization-induced numerical dissipation due to insufficient horizontal resolution; and too much parameterized dissipation. 


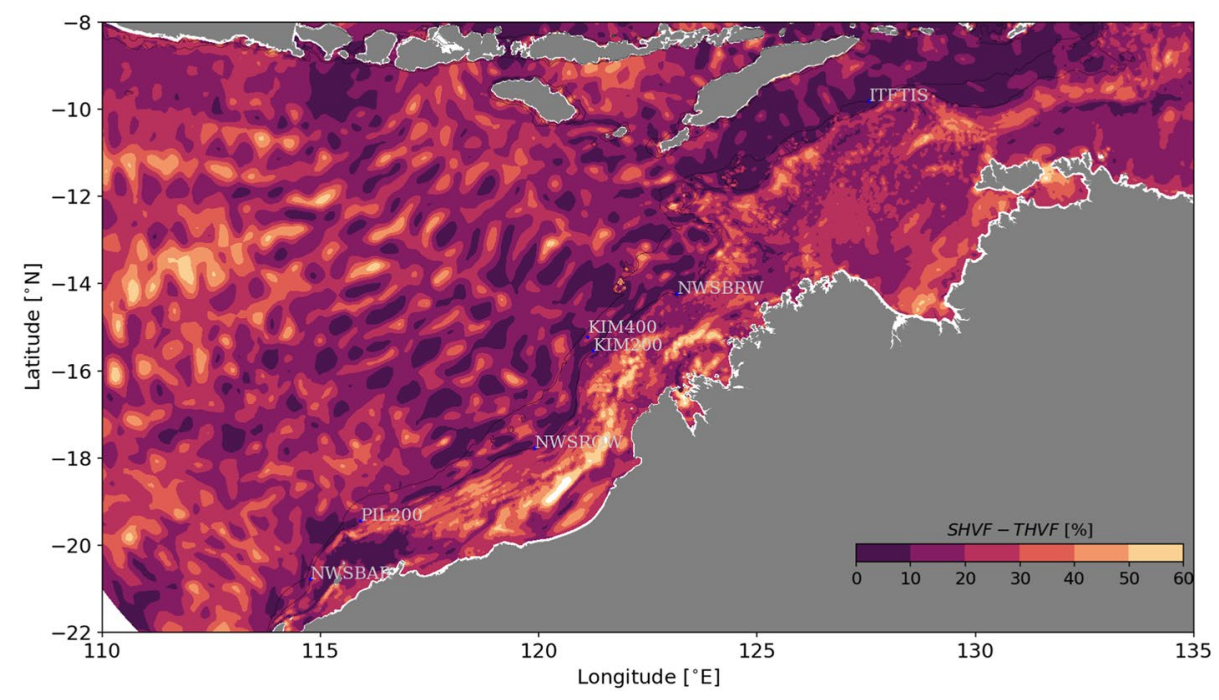

Figure 10. Difference between SHVF (Equation 8) and THVF (Equation 9). Here, a small number means the seasonal harmonic model does not improve the predictive skill appreciably.

\subsection{Amplitudes of Major Tidal Frequencies and Some Seasonal Sidelines}

Spatial variations in the mean $M_{2} S S H_{B C}$ amplitude revealed several hot spot regions, including around the major Indonesian Straits (Lombok, Ombai, Timor), on the NWS near Rowley Shoals, and the Browse Basin regions. The model also revealed vast regions of standing wave-like characteristics throughout the domain, including on the shelf between the 200-m and 500-m isobaths (Figure 13a). The standing wave patterns led to nodes and antinodes in $S_{S H} H_{B C}$ separated by spatial scales of roughly one internal tide wave length (roughly $50 \mathrm{~km}$ on the shelf and $100 \mathrm{~km}$ in the deep basin). The $K_{1}$ component, dominant in the Timor Sea but weak on the North West Shelf, also formed standing internal tide patterns (Figure 13b). Qualitatively, this agreed with the spatial variations from the altimetry-derived HRET model (Figure 1). Some obvious differences between the HRET and the SUNTANS-derived harmonic amplitudes were close to islands (e.g., Lombok Strait) and on the NWS in depths $<500 \mathrm{~m}$. A known limitation of the satellite altimetry processing is the necessity to filter out signals in shallow water where the internal tides and barotropic tides vary over similar length scales (e.g., Zaron, 2019). The modeled $K_{1}$ amplitude was also consistently larger than the HRET $K_{1}$ amplitude (not shown).

The six seasonal sideline harmonic amplitudes of the $M_{2}$ frequency exhibited a qualitatively similar spatial structure to $M_{2}$ albeit with roughly $30 \%$, or smaller, amplitude (Figure 14). The annual modulates $\left(M_{2} \pm \omega_{A}\right.$, also referred to as $M A_{2}$ and $M B_{2}$ in Zaron, 2019) were generally largest, particularly on the NWS, although the semiannual and triannual sidelines were large, exceeding $2 \mathrm{~cm}$, throughout the Indo-Australian Basin

Table 3

Validation Metrics for the SUNTANS-Derived $\mathrm{SSH}_{B C}$ From Equation 6 Compared Against In-Situ Observations

\begin{tabular}{lccr}
\hline Site & Dates & RMSE $(\mathrm{cm})$ & Skill \\
\hline ITFTIS & July 2013 to June 2014 & 3.25 & 0.47 \\
KIM200 & July 2013 to June 2014 & 2.29 & 0.37 \\
KIM400 & July 2013 to June 2014 & 1.82 & -0.60 \\
PIL200 & July 2013 to June 2014 & 2.85 & 0.02 \\
NWSBAR & August 2019 to February 2020 & 4.58 & 0.12 \\
NWSROW & August 2019 to February 2020 & 2.86 & 0.45 \\
NWSBRW & August 2019 to February 2020 & 5.80 & 0.67 \\
\hline
\end{tabular}

and around the Indonesian Archipelago. The HRET $M A_{2}$ and $M B_{2}$ were globally significant in the region directly south of Lombok Strait although were about $50 \%$ smaller than the SUNTANS-derived amplitudes (not shown). This discrepancy may be due to the longer record length used to derive the HRET amplitudes. We quantify the contributions of these seasonal terms to the total signal variance in the next section. Again, the decorrelation length scale for the peak amplitudes in any given seasonal sideline harmonic was of the order of one internal tide wavelength. This explains the variability in the observed amplitudes at each of these frequencies (see Figure 8). For example, at PIL200, the modeled amplitude of $M_{2}-\omega_{A}$ was $0.7 \mathrm{~cm}$ whereas $M_{2}+\omega_{A}$ was $0.3 \mathrm{~cm}$. There were, however, regions within one wave length (roughly $50 \mathrm{~km}$ ) where $M_{2}+\omega_{A}$ exceeds $0.5 \mathrm{~cm}$ (Figure 14a). Similar examples exist for the other sites where peaks in specific seasonal harmonics were locally specific. For example, the $\left|\eta_{M 2}\right|$, reconstructed from the seasonal harmonics, peaked at 


\begin{tabular}{|c|c|c|c|c|c|c|c|}
\hline Site & $\left\langle S S H_{B C}\right\rangle^{2}\left[\mathrm{~cm}^{2}\right]$ & SHVF & THVF & $V F_{M 2}$ & $V F_{K 1}$ & $S V F_{M 2}$ & $S V F_{K 1}$ \\
\hline ITFTIS & 14.4 & 94.7 & 87.2 & 2.1 & 61.8 & 40.3 & 9.5 \\
\hline KIM200 & 5.9 & 68.0 & 54.3 & 54.6 & 9.9 & 20.7 & 30.5 \\
\hline KIM400 & 3.1 & 49.7 & 28.5 & 67.2 & 6.8 & 40.4 & 36.4 \\
\hline PIL200 & 2.5 & 61.7 & 35.5 & 43.2 & 23.8 & 59.1 & 16.4 \\
\hline NWSBAR & 3.0 & 68.4 & 58.3 & 76.9 & 11.9 & 10.4 & 23.2 \\
\hline NWSROW & 4.5 & 75.7 & 62.8 & 36.6 & 8.0 & 14.0 & 28.1 \\
\hline NWSBRW & 33.2 & 94.5 & 89.7 & 56.9 & 2.9 & 2.1 & 15.4 \\
\hline
\end{tabular}

KIM200 in January, whereas peaks at KIM400 lagged KIM200 by about 2 months and were about $20 \%$ of the amplitude (see Figures $5 \mathrm{~b}$ and $6 \mathrm{~b}$ ). It is the spatial variations in phase, not just the amplitude, of these seasonal sideline harmonics that generates the complicated spatiotemporal variability observed between KIM200 and KIM400. These effects need to be considered when attempting to interpret the broader regional seasonal variability from single point observations.

\subsection{Importance of Seasonal Harmonics and Standing Internal Tides}

Internal tides at a fixed site, like a mooring, are a result of the barotropic forcing over nearby topography and remotely generated internal waves propagating long distances. Although the dominant local barotropic tidal harmonics are likely to be present in the observed internal wave response, multiple generation sites and long propagation distances lead to
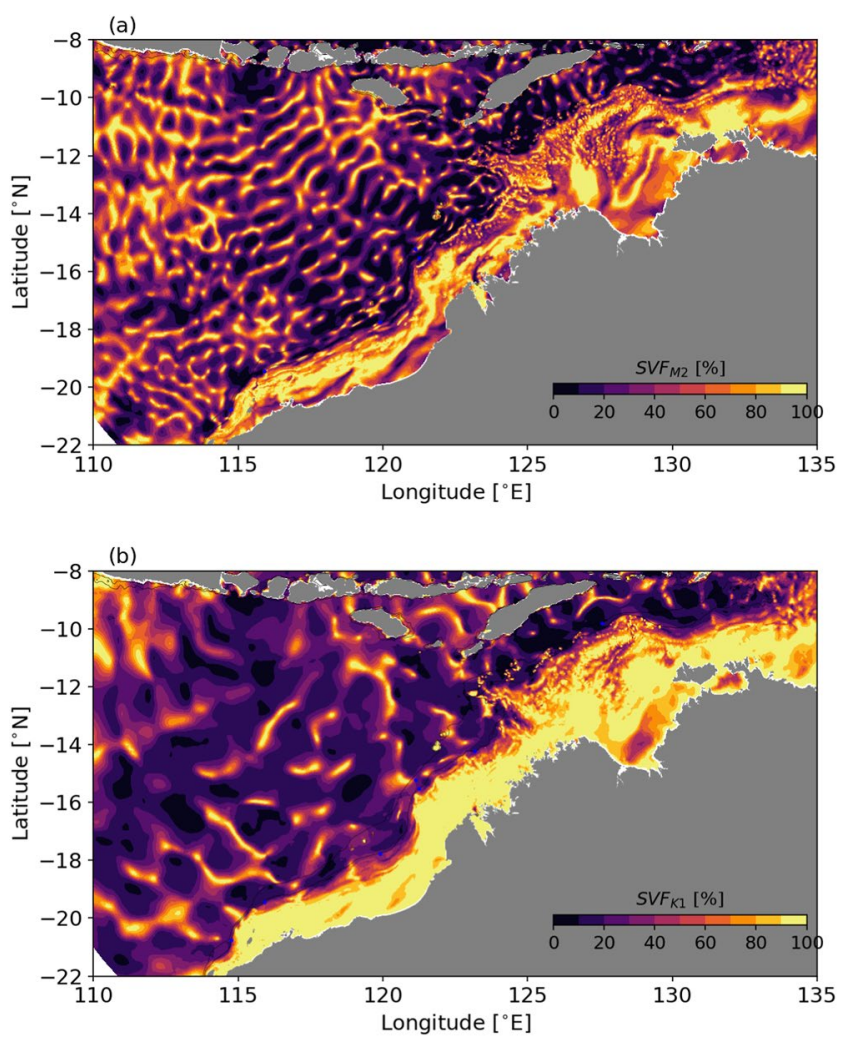

Figure 11. Percentage of variance of the SUNTANS baroclinic SSH $\left(S V F_{m}\right.$, Equation 11) explained by the seasonal harmonics in the (a) $M_{2}$ and (b) $K_{1}$ bands. high spatial variability in the frequency content of ocean scalars. For example, despite the $M_{2}$ barotropic tide being dominant on the NWS (Holloway, 1983), the KIM200 mooring was the only site where the $M_{2}$ baroclinic component was dominant throughout the year (see Figure 5). This is contrary to the conventional view that the $M_{2}$ internal tide is dominant (e.g., Holloway, 2001; Kelly et al., 2014; Rayson et al., 2012). While this conventional picture was generally true in the numerical model solution (see e.g., Figure 13), the results presented here indicate that large spatial variations in amplitude can occur over short distances of generally less than one wave length. Conversely in the Timor Sea, there is a $M_{2}$ tidal amphidrome, resulting in the dominance of diurnal barotropic tides (see e.g., Robertson \& Ffield, 2008). Based on analysis of the ITFTIS mooring, it may be tempting to conclude that the $K_{1}$ internal tide is therefore also dominant in the Timor Sea. The regional internal tide model highlighted, however, that there are regions within $30 \mathrm{~km}$ of the mooring where the $M_{2}$ component is actually dominant (Figure 12a), likely due to remotely generated internal tides. This high spatial variability is thus an important consideration to take into account when either choosing mooring field sampling strategies to study the internal tide or when interpreting data from a mooring.

The seasonal node/antinode variability is the main reason why the KIM200 and KIM400 sites have such a different internal tide variability, despite being relatively close in space. Model results indicated that the KIM400 mooring was located in an $M_{2}$ node region throughout the year (Figure 15), whereas the KIM200 was in a node for only part of the year (e.g., October), and in an antinode during January. This spatial feature in the model-derived $S_{S} H_{B C}$ corresponded with the in-situ data: the $M_{2}$ amplitude at KIM200 was lowest in October and highest in January in 

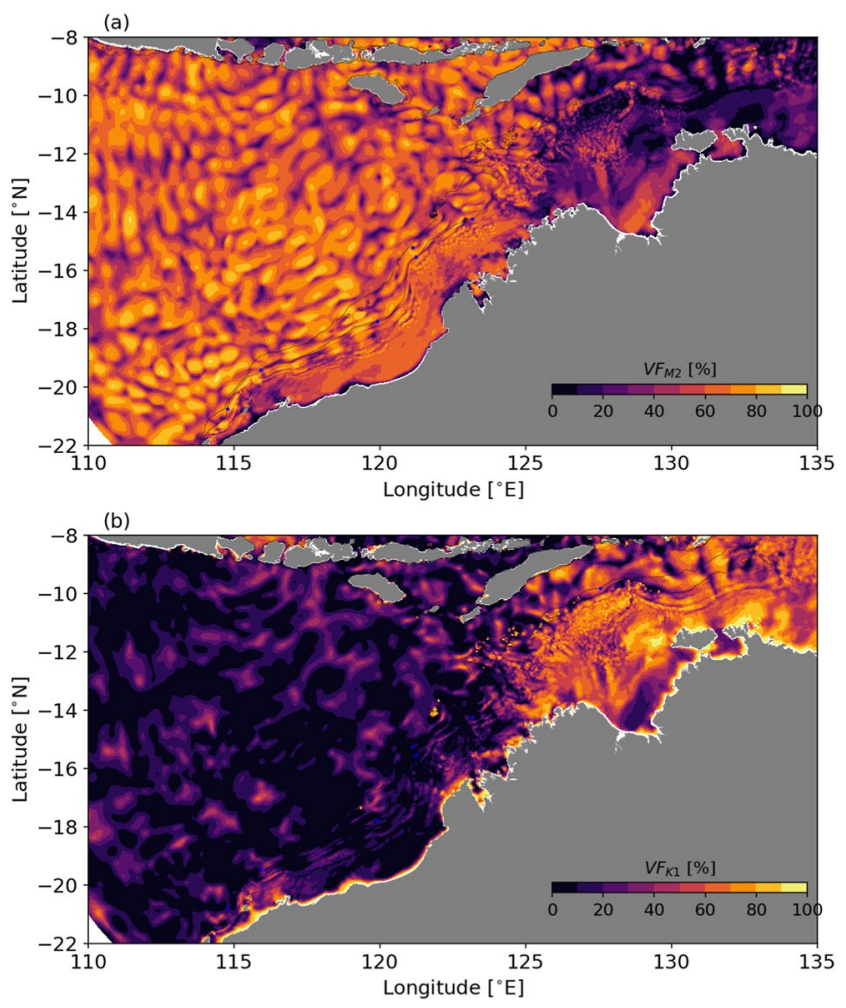

Figure 12. Percentage of variance of the SUNTANS baroclinic SSH $\left(V F_{m}\right.$, Equation 10) explained by the (a) $M_{2}$ and (b) $K_{1}$ band harmonics, i.e., including the seasonal harmonics. both years of the observation record (Figure 5a); whereas the opposite occurred at KIM400 (Figure 6a). Note that the baroclinic velocity will have the opposite response to $S_{S H}$ C the velocity will peak in the $S S H_{B C}$ nodes and be smallest in the antinodes (see e.g., Rayson et al., 2012). Variations in standing wave locations over a year, not changes in the tidal forcing or wave dissipation, was therefore deemed to be a dominant driver of the observed seasonal internal tide amplitude modulation at the shelf sites. It demonstrates that the observed internal tide amplitude is not directly correlated with the magnitude of the local tidal forcing.

\subsection{Overview of Regional Variability}

The key results from the 12-month ocean model seasonal harmonic fit were:

1. Internal tide amplitudes in all frequency bands exhibited standing wave patterns

2. Predictability at a given observation point generally coincided with the location of nodes and antinodes and also with the total signal variance fraction in the seasonal harmonics

3. The ability of both the seasonal and tidal harmonic models to capture the total $S S H_{B C}$ signal variance was regionally dependent, with the Timor Sea being the most predictable and the Pilbara region (southern NWS) being the least predictable

4. Overall, the seasonal model captured more of the signal variance, as it includes seven times more frequencies and hence amplitude parameters than the tidal model

5. In regions where the harmonic fit described zero percent of the variance, like on the shelf in water shallower than $200 \mathrm{~m}$, a seasonal model fit is essential

Finally, we have not presented any analysis of internal tide-induced (baroclinic) velocity perturbations. It should be noted that in places where standing internal waves are dominant (almost everywhere in this domain), regions of small $S_{S} H_{B C}$, or isotherm displacement amplitude, variance will likely have large baroclinic velocity variance. Interpretation of individual and isolated in-situ observations requires knowledge of the broader spatial context, namely these regional internal tide interference patterns.

\section{Drivers of Seasonal Internal Tide Variability}

The 12-month ocean model results have shown that the Indo-Australian Basin, spanning 110-115 E and 15-10 S, was a region where seasonal harmonics contribute significantly to the total signal variance (e.g., Figures 10 and 14). It was also a region where the residual variance of the seasonal harmonic model was relatively large (Figure 9b). Intermittent internal tides that lead to nonlinear spectral broadening and enhanced seasonal harmonics are primarily believed to be caused by perturbations in the internal wave phase speed due to time-variable ocean properties, such as in the stratification, mean flow, and relative vorticity (Zaron \& Egbert, 2014). Savage et al. (2020) proposed two parameterizations for the energy removed from the internal tide at the forcing frequencies by the background ocean processes. The first scheme accounts for advection by the background flow, the second accounts for refraction by variations in stratification. They demonstrated that the energy lost due to refraction was greater than advection in the Tasman Sea. Kelly et al. (2021) computed the energy lost from the $M_{2}$ internal tide on a global scale and demonstrated that the Indo-Australian Basin was a regional hot spot for this refractive process. Note that energy is not lost completely; it is transferred to other frequencies. Here we look for evidence that refraction due to stratification (and phase speed) variations modifies the $M_{2}$ internal tide amplitude in our model results. 

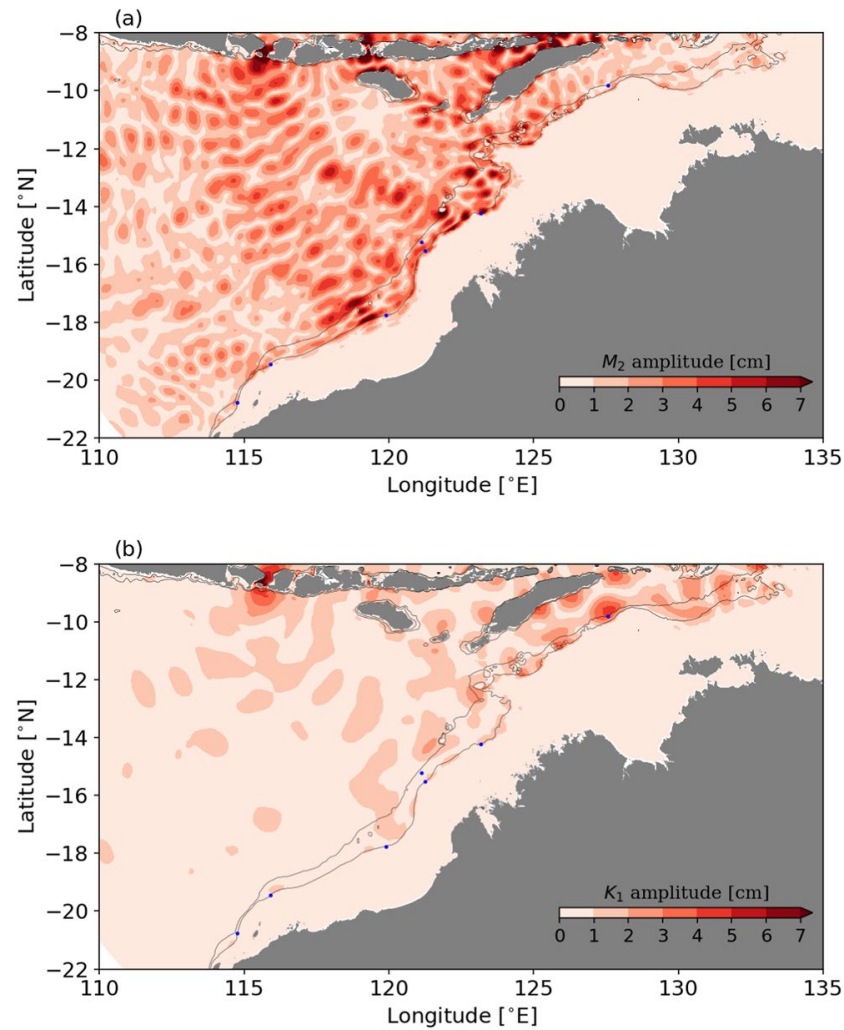

Figure 13. Mean baroclinic sea surface height harmonic amplitudes for (a) the $M_{2}$ and (b) the $K_{1}$ tidal constituents from the 12-month SUNTANS simulation.

The dominant mesoscale flow feature in the NE Indian Ocean is the strong but seasonally varying Indonesian Throughflow (ITF) that flows in a westerly direction from roughly June to December (Meyers et al., 1995). This flow breaks down into a series of eddies (instabilities) between December and March when the monsoon winds shift from south easterly to northwesterly (Feng \& Wijffels, 2002). Monthly averaged $S S H$ and surface currents from the internal tide-resolving SUNTANS model exhibited these features. In particular, a large N-S SSH gradient and strong westward surface flow was present around October 2013 (Figure 16a). Whereas by January 2013, the large-scale mean N-S SSH gradient had relaxed and was replaced by a series of geostrophically balanced regional mesoscale eddies (Figure 16b). The mode-1 linear phase speed (Zhao et al., 2016)

$$
c_{1}=\frac{\omega}{\left(\omega^{2}-f^{2}\right)^{1 / 2}} c
$$

where $f$ is the Coriolis frequency and $c$ is given by the normal mode eigenvalue problem, was calculated using only the background stratification for each month. The phase speed was up to $0.3 \mathrm{~m} \mathrm{~s}^{-1}$ faster in the austral summer compared with spring (February minus October) over the NWS and south of Indonesia (Figure 16c). In the deeper regions of the Indo-Australian Basin, the phase speed was slower by about $0.1 \mathrm{~m}$ $\mathrm{s}^{-1}$ in summer compared with spring, although this was in a region where the mean total phase speed was greater than $3.0 \mathrm{~m} \mathrm{~s}^{-1}$.

To identify the temporal modulation of the internal tide amplitude between Indonesia and the NWS due to refraction and/or advection, we interpolated $\left|\eta_{M_{2}}(t)\right|$ along a line between Lombok Strait and the Rowley Shoals (transect line shown in Figure 16c). Seasonal variations in $\left|\eta_{M 2}(t)\right|$ were evident at each location along the transect; e.g., along $10.5 \mathrm{~S}$ there were two major peaks in $\left|\eta_{M 2}(t)\right|$, one in January and one in June 2014. Conversely, along $11 \mathrm{~S}$ there was a single peak around February 2014. These differences in seasonal peaks over such short distances can partly be explained by variations in arrival time due to changes in phase 

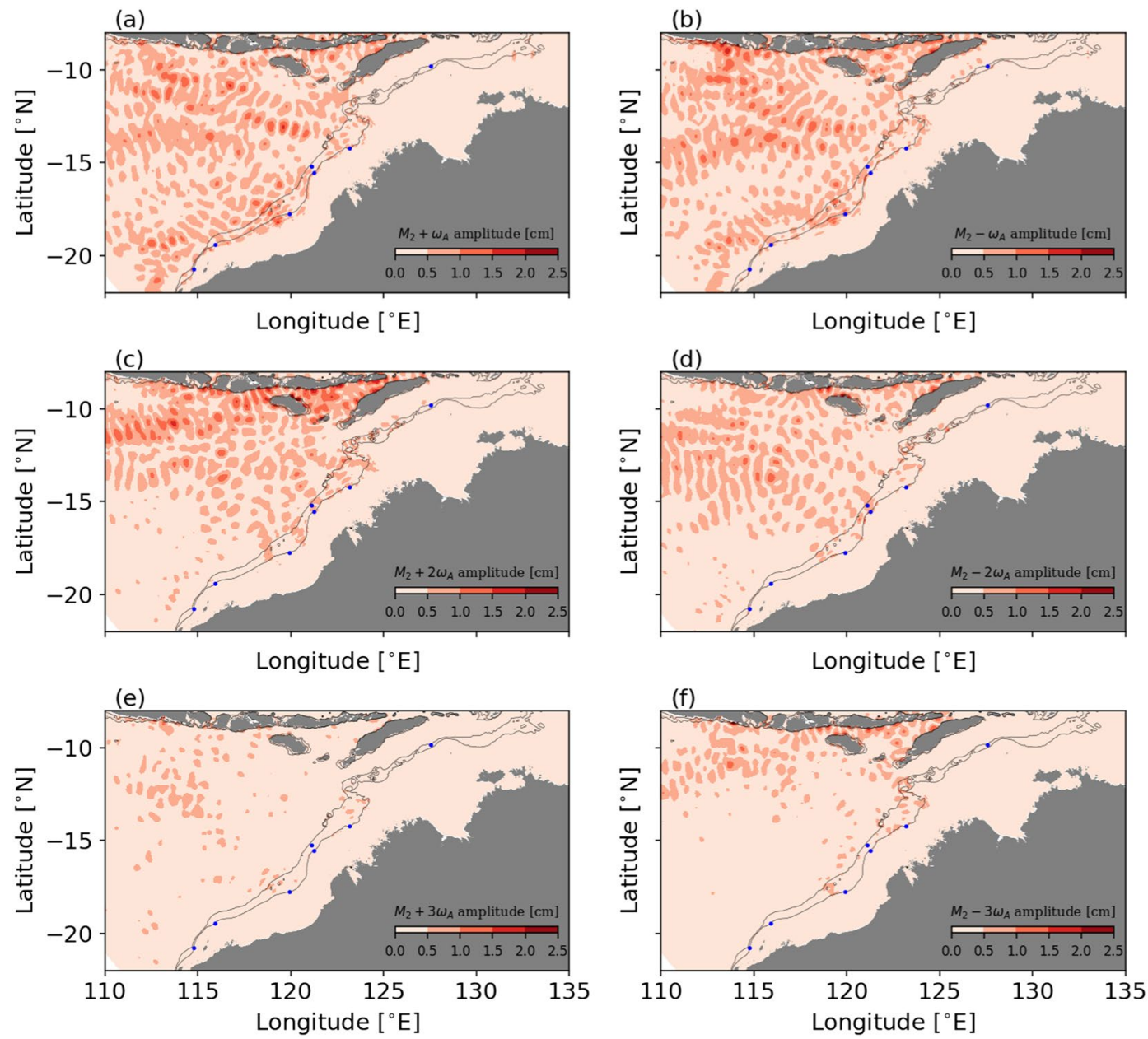

Figure 14. Harmonic amplitudes of the six $M_{2}$ annual modulates.

speed (Figure 17b). Assuming for simplicity that wave propagation is one-dimensional, we calculated the propagation time ${ }^{t}$ ) from the time-varying phase speed along the transect line as

$$
\tau(y, t)=\int_{0}^{y} \frac{1}{c_{1}\left(y^{\prime}, t\right)} d y^{\prime},
$$

where $y$ is the distance along the transect line. Contours of $\tau(y, t)$ help identify the drivers of spatial differences in $\left|\eta_{M 2}(t)\right|$ due to stratification-induced refraction (black contours on Figure 17). The peak in $\left|\eta_{M 2}(t)\right|$ at $11^{\circ} \mathrm{S}$ during March 2014 corresponded with a period when the line of constant propagation time migrated further south due to the increased phase speed south of Lombok Strait. Lines of constant propagation time were less indicative of amplitude modulations further from the primary internal tide source regions, e.g., between $12^{\circ} \mathrm{S}$ and $16 \mathrm{~S}$ in Figure 17a. This discrepancy is likely due to other processes causing perturbations in the mode- 1 phase speed (namely the mean flow and vorticity), and also due to the wave propagation being two-dimensional.

Evidence of energy removal from the primary internal tide frequencies was inferred by finding time periods when the signal amplitude was reduced along propagation paths. To visualize the amplitude reduction in the direction of wave propagation, we performed a directional decomposition of the complex harmonic amplitudes using the technique outlined in Gong et al. (2021). This technique takes a 2D Fourier transform of the complex spatial internal tide amplitude, filters the horizontal wavenumbers (that are both positive and negative) according to a directional band of choice, and then takes the inverse Fourier transform. Note that this method differs from the explicit plane-wave fitting technique outlined in Zhao et al. (2016) in that 

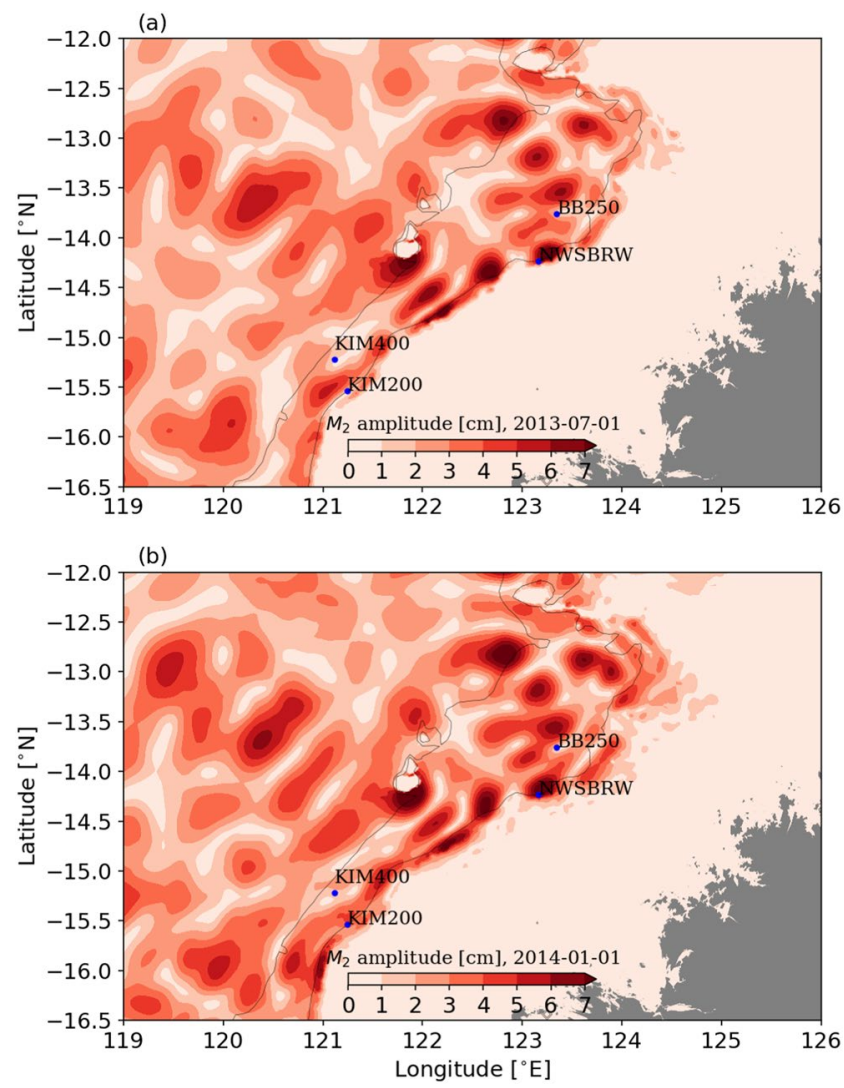

Figure 15. Snapshots of $\left|\eta_{M 2}(t)\right|$ from the Browse Basin region during July and January.

the Gong et al. (2021) method does not require a priori specification of the horizontal wavenumber, or the number of waves to include. It does, however, require a gridded amplitude field and so is suited to numerical model output.

The directional decomposition revealed the SE propagating component (filter band of 0-90 $\mathrm{CCW}$ from E) originated from Indonesia, while the NW propagating component was the NWS-generated internal tide (Figure 18). Temporal modulations of the SE component were most pronounced in the center of the Indo-Australian Basin and on the NWS slope. The modulating component on the NWS was evident in the multidirectional signal (e.g., Figure 11), and is described in Rayson et al. (2012). Between $8^{\circ} \mathrm{S}$ and $14 \mathrm{~S}$, the SE propagating component, which originated near Lombok strait, was 1-2 cm (50\% or more) smaller during October than it was during February (Figures 18d-18f). Likewise, the NW propagating component, which originated along the NWS-break, was 1-2 cm smaller near Indonesia during October than it was in February (Figures 18a-18c). We speculate from this analysis that the amplitude reduction in $\mathrm{SSH}_{B C}$ around October was caused by refraction of the low-mode internal tide by the changes in phase speed due to the strong ITF during this period (e.g., Figure 16a). Scattering of energy from internal waves into the background mean flow can cause some of the amplitude variations (e.g., Dunphy \& Lamb, 2014), thus it is difficult to attribute seasonal internal tide amplitude variations to a single process without a proper decomposition of the model flow field into mean and wave-induced flow.

\section{Conclusions}

A key output from harmonically decomposing the internal tide amplitude or $S S H_{B C}$ from primitive equation ocean model solutions, like our one-year SUNTANS solution for the Indo-Australian Basin, is a climatological database of $S S H_{B C}$ harmonic amplitudes. By including the "seasonal sidelines" in our harmonic analysis, as suggested in Arbic et al. (2015), we have shown that a larger amount of internal tide variance is captured, particularly around the shelf regions (see e.g., Figure 10 and Table 4). This description of the cli- 


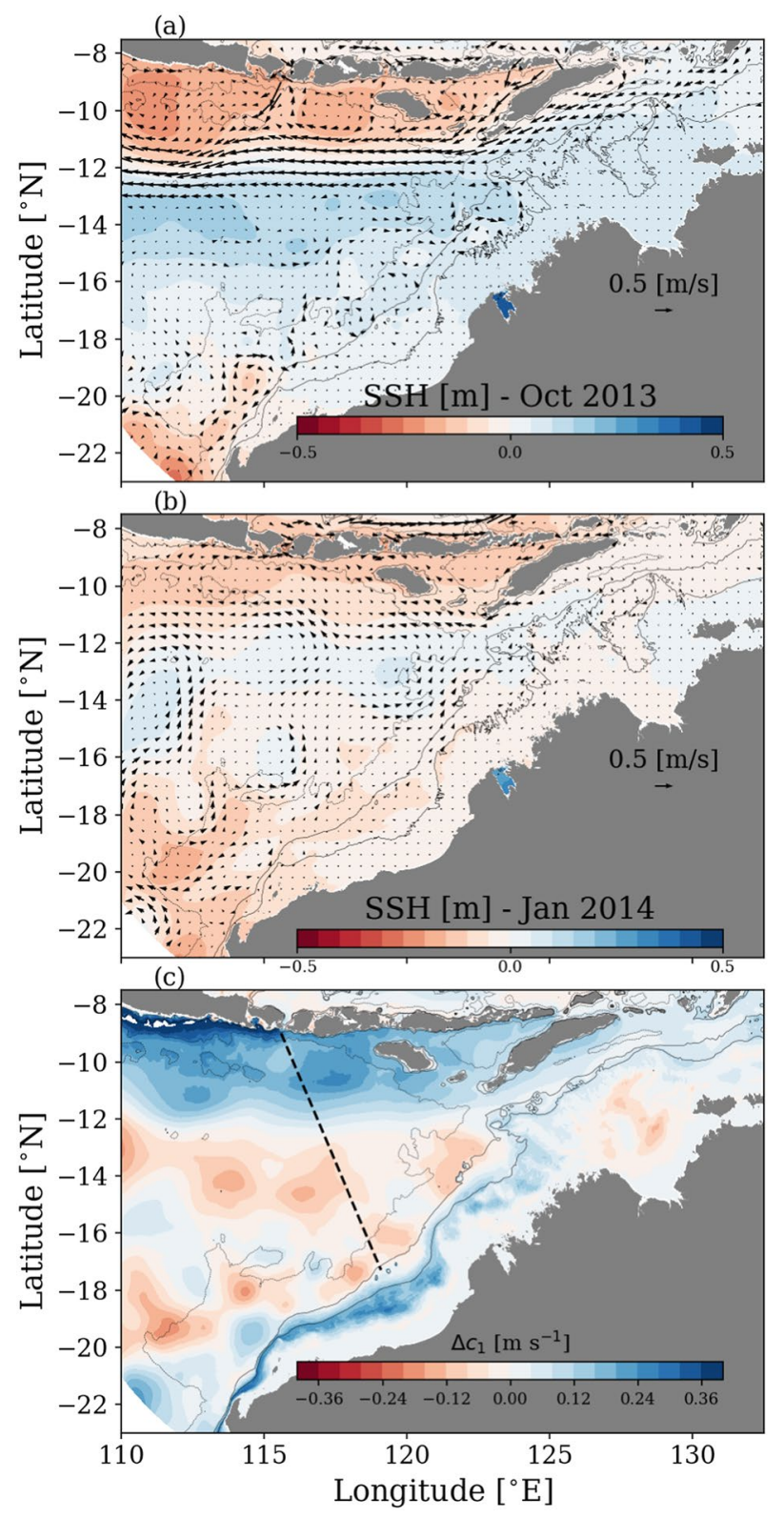

Figure 16. Monthly averaged sea surface height (contours) and surface velocity (vectors) from the SUNTANS model for (a) October 2013 and (b) January 2014. The vector scale is indicated in the bottom right corner of each panel. (c) Indicates the mode-1 linear phase speed difference between the two months (January minus October).

matology, and any future improvements that account for interannual variability, has a number of practical applications, including: allowing the partial removal of the internal tide signal in future satellite altimetry data sets (e.g., Morrow et al., 2019); and supplying boundary conditions for regional internal wave modeling applications (e.g., Gong et al., 2021). Here, we have constructed a regional internal tide database of $S S H_{B C}$ amplitude parameters, and a similar global database could readily be calculated using output from a global internal tide-resolving model, e.g., the $1 / 25^{\circ} \mathrm{HyCOM}$ model in Savage et al. (2017) or the $1 / 48^{\circ}$ LLC4320 MITGCM run in Torres et al. (2018).

Various studies, including this one, have demonstrated that tidal harmonics are a useful description of internal tide variability when applied over short-time periods (generally less than a month). The most important aspect of internal tide prediction is how to model the temporal modulation of these short-time window amplitudes. Here, we used a seasonal harmonic model that was motivated by the modulation of the Timor 


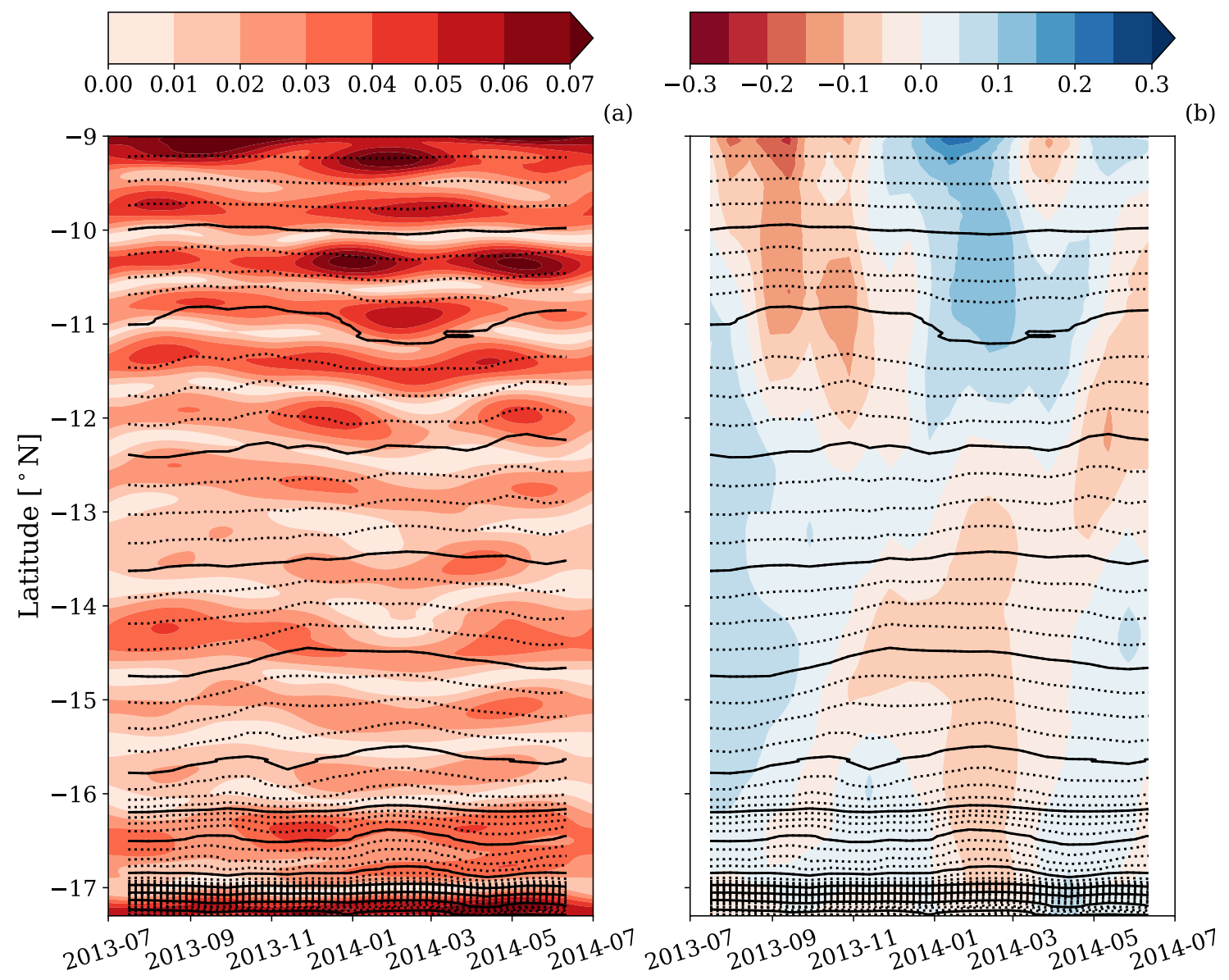

Figure 17. Temporal evolution of (a) $\left|\eta_{M 2}\right|(\mathrm{m})$ and (b) mode-1 phase speed difference between summer and spring (m/s) along the transect in Figure 16c. Black contours indicate an estimate of the propagation time from the northernmost point in one cycle intervals (dotted contours indicate quarter cycle intervals).

Sea internal tides, primarily driven by seasonal changes in the ocean stratification (Kelly et al., 2014). This seasonal model is less suited to other regions on the globe, the PIL200 site is one example, where internal tide variations are due to more transient (aperiodic) features like mesoscale eddies.

To model the temporal modulation in these regions with aperiodic features, nonparametric techniques like splines or Gaussian processes are likely to be better suited (see Sarkar et al., 2018). These nonparametric methods, however, rely on having recent data to make predictions of internal tides into the short-term future. In regions with no data, they would fall back to a deterministic mean function to make predictions, e.g., a harmonic model. Another approach is to regress the amplitude with an external forcing variable, as Matte et al. (2013) did to model surface tides in an estuary where they used river stage as the external variable. Their approach is attractive as it captures nonstationarity; however, the challenge with predicting internal waves is finding a suitable (and observable) external variable that correlates with the amplitude. More theoretical work is required. In regions where internal tide prediction is important for operational decision making, these data-driven techniques will be necessary. Our parametric seasonal harmonic model provided a better prediction of the internal tides throughout most of the study region, and thus is a useful starting point for more data-intensive statistical modeling techniques like Sarkar et al. (2018), that is, as a suitable mean function. Last, our method aids in identification of regions where the internal tides are intermittent and therefore could be targeted in future field campaigns. 
(a)

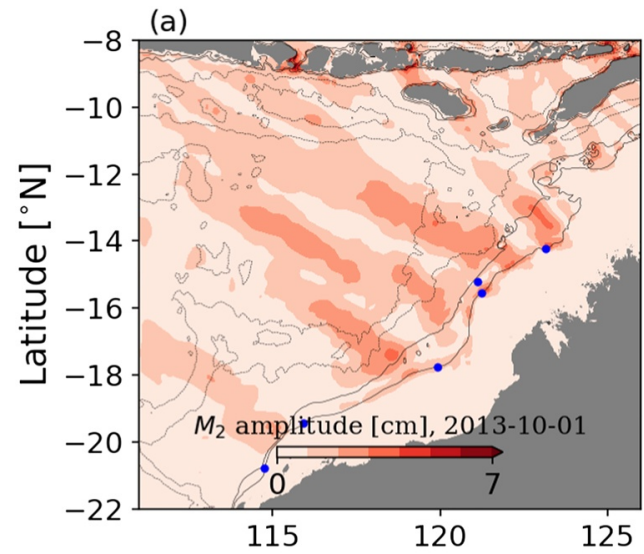

(d)

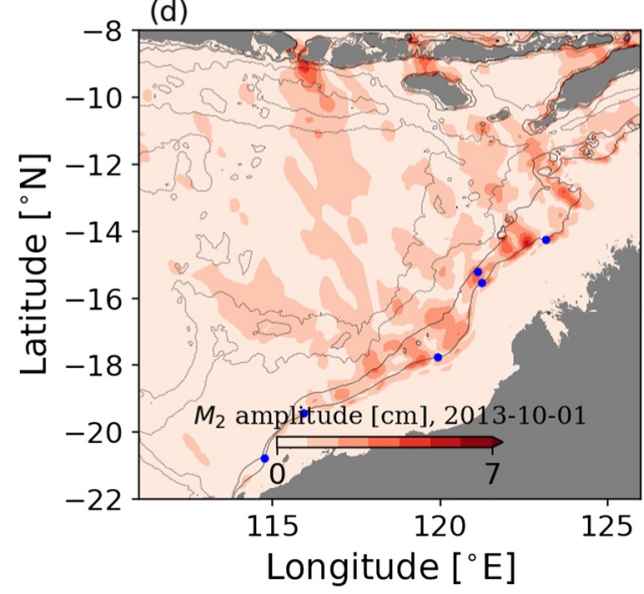

(b)

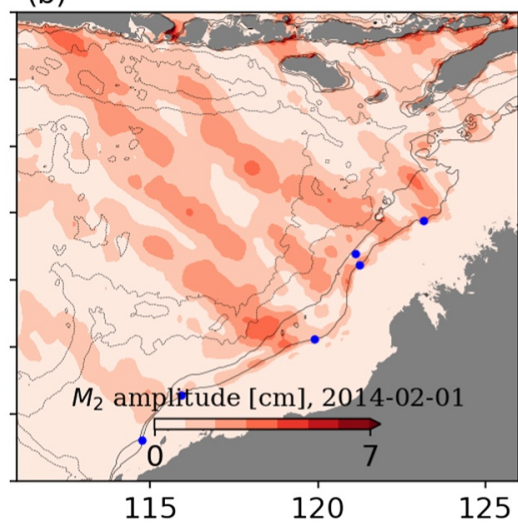

(e)

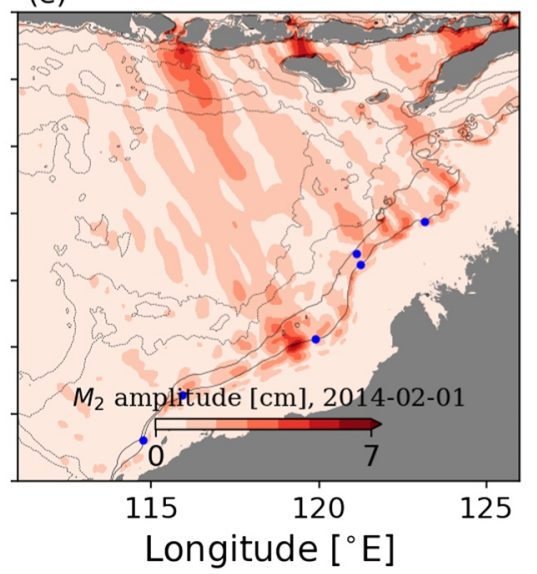

(c)

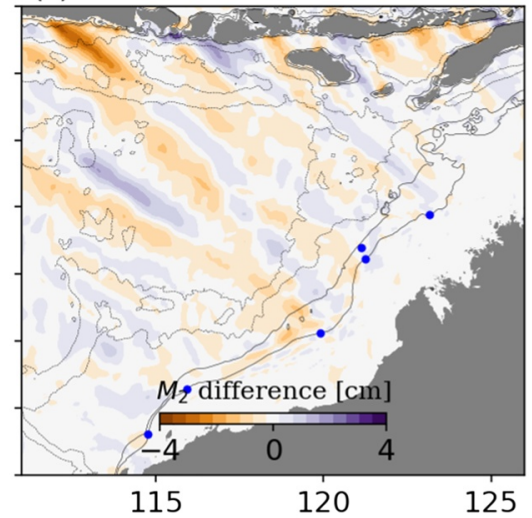

(f)

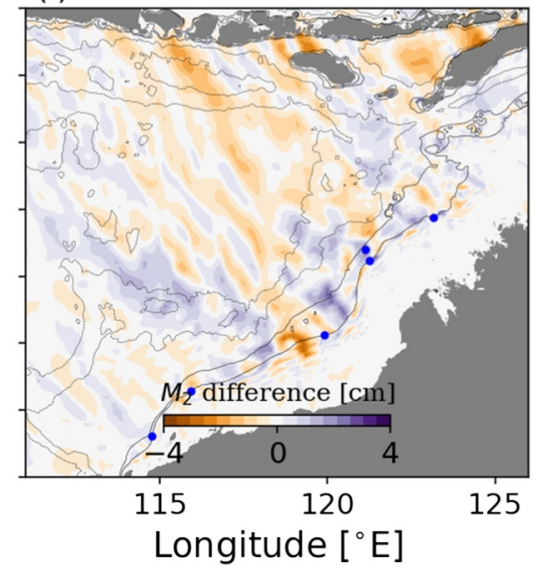

Figure 18. Directionally decomposed internal tide sea surface height amplitude, $\eta_{M 2}(t)$, for (a and d) October 2013 and (b and e) February 2014 . The top row indicates the NW propagating portion of the signal, whereas the bottom row indicates the SE component. The last column shows the difference between October and February for (c) the NW and (f) the SE components.

\section{Acknowledgments}

This work was supported by the Australian Research Council Industrial Transformation Research Hub for Offshore Floating Facilities (IH140100012). This work was supported by resources provided by the Pawsey Supercomputing Centre with funding from the Australian Government and the Government of Western Australia. We thank two anonymous reviewers for their helpful comments and suggestions that improved earlier versions of this paper.

\section{Data Availability Statement}

Data were sourced from Australia's Integrated Marine Observing System (IMOS)_-IMOS is enabled by the National Collaborative Research Infrastructure Strategy (NCRIS). It is operated by a consortium of institutions as an unincorporated joint venture, with the University of Tasmania as Lead Agent. Quality controlled observation data are available on the Australian Ocean Data Network (https://portal.aodn.org.au). Numerical model tide data are available at: https://www.tpxo.net/regional. Initial and boundary condition data are at https://resources.marine.copernicus.eu/documents/PUM/CMEMS-GLO-PUM-001-030.pdf. Processed numerical model output and observation data are archived on the UWA library research repository (https:// doi.org/10.26182/8jx9-m532). Model setup files to reproduce the raw model output can be obtained by contacting the author.

\section{References}

Arbic, B. K., Lyard, F., Ponte, A., Ray, R. D., Richman, J. G., Shriver, J. F., et al. (2015). Tides and the SWOT mission: Transition from Science Definition Team to Science Team. Civil and Environmental Engineering Faculty Publications and Presentations, 336, 1-8.

Bachman, S. D., Shakespeare, C. J., Kleypas, J., Castruccio, F. S., \& Curchitser, E. (2020). Particle-based lagrangian filtering for locating wave-generated thermal refugia for coral reefs. Journal of Geophysical Research: Oceans, 125, e2020JC016106. https://doi. org/10.1029/2020JC016106

Buijsman, M. C., Arbic, B. K., Richman, J. G., Shriver, J. F., Wallcraft, A. J., \& Zamudio, L. (2017). Semidiurnal internal tide incoherence in the equatorial Pacific. Journal of Geophysical Research: Oceans, 122, 5286-5305. https://doi.org/10.1002/2016JC012590

Cartwright, D. E., \& Tayler, R. J. (1971). New computations of the tide-generating potential. Geophysical Journal of the Royal Astronomical Society, 23(1), 45-73. https://doi.org/10.1111/j.1365-246X.1971.tb01803.x 
Castaño-Tierno, A., Mohino, E., Rodríguez-Fonseca, B., \& Losada, T. (2018). Revisiting the CMIP5 thermocline in the equatorial pacific and Atlantic Oceans. Geophysical Research Letters, 45, 12963-12971. https://doi.org/10.1029/2018GL079847

Colosi, J. A., \& Munk, W. (2006). Tales of the venerable Honolulu tide gauge. Journal of Physical Oceanography, 36(6), 967-996. https://doi.org/10.1175/JPO2876.1

Dee, D. P., Uppala, S. M., Simmons, A. J., Berrisford, P., Poli, P., Kobayashi, S., et al. (2011). The ERA-interim reanalysis: Configuration and performance of the data assimilation system. Quarterly Journal of the Royal Meteorological Society, 137(656), 553-597. https://doi. $\operatorname{org} / 10.1002 / q j .828$

Doodson, A. T. (1921). The harmonic development of the tide-generating potential. Proceedings of the Royal Society of London-Series A: Containing Papers of a Mathematical and Physical Character, 100(704), 305-329. https://doi.org/10.1098/rspa.1921.0088

Dunphy, M., \& Lamb, K. G. (2014). Focusing and vertical mode scattering of the first mode internal tide by mesoscale eddy interaction. Journal of Geophysical Research: Oceans, 119, 523-536. https://doi.org/10.1002/2013JC009293

Dushaw, B. D., Worcester, P. F., \& Dzieciuch, M. A. (2011). Deep-Sea Research I On the predictability of mode-1 internal tides. Deep-Sea Research Part I, 58(6), 677-698. https://doi.org/10.1016/j.dsr.2011.04.002

Egbert, G., \& Erofeeva, S. (2002). Efficient inverse modeling of barotropic ocean tides. Journal of Atmospheric and Oceanic Technology, 19, 183-204. https://doi.org/10.1175/1520-0426(2002)019<0183:eimobo>2.0.co;2

Egbert, G., \& Ray, R. (2017). Tidal prediction. Journal of Marine Research, 75(3), 189-237. https://doi.org/10.1357/002224017821836761

Fairall, C. W., Bradley, E. F., Hare, J. E., Grachev, A. A., \& Edson, J. B. (2003). Bulk parameterization of air-sea fluxes: Updates and verification for the COARE algorithm. Journal of Climate, 16(4), 571-591. https://doi.org/10.1175/1520-0442(2003)016<0571:bpoasf >2.0.co;2

Feistel, R. (2008). A Gibbs function for seawater thermodynamics for -6 to $80^{\circ} \mathrm{C}$ and salinity up to $120 \mathrm{~g} \mathrm{~kg}^{-1}$. Deep-Sea Research Part I: Oceanographic Research Papers, 55, 1639-1671. https://doi.org/10.1016/j.dsr.2008.07.004

Feng, M., \& Wijffels, S. (2002). Intraseasonal variability in the SEC of the East Indian ocean. Journal of Physical Oceanography, 32, 265277. https://doi.org/10.1175/1520-0485(2002)032<0265:ivitse >2.0.co;2

Foreman, M. G. G. (1977). Manual for tidal heights analysis and prediction (Tech. Rep.). Patricia Bay: Institute of Ocean Sciences.

Fringer, O. B., Gerritsen, M., \& Street, R. L. (2006). An unstructured-grid, finite-volume, nonhydrostatic, parallel coastal ocean simulator. Ocean Modelling, 14(3-4), 139-173. https://doi.org/10.1016/j.ocemod.2006.03.006

Gong, Y., Rayson, M. D., Jones, N. L., \& Ivey, G. N. (2019). The effects of remote internal tides on continental slope internal tide generation. Journal of Physical Oceanography, 49(6), 1651-1668. https://doi.org/10.1175/jpo-d-18-0180.1

Gong, Y., Rayson, M. D., Jones, N. L., \& Ivey, G. N. (2021). Directional decomposition of internal tides propagating from multiple generation sites. Ocean Modelling, 162, 101801. https://doi.org/10.1016/j.ocemod.2021.101801

Holloway, P. E. (1983). Tides on the Australian North-West shelf. Marine and Freshwater Research, 34(1), 213-230. https://doi.org/10.1071/ MF9830213

Holloway, P. E. (2001). A regional model of the semidiurnal internal tide on the Australian North West Shelf. Journal of Geophysical Research, 106(C9), 19625-19638. https://doi.org/10.1029/2000JC000675

Huess, V., \& Andersen, O. B. (2001). Seasonal variation in the main tidal constituent from Altimetry. Geophysical Research Letters, 28(4), 567-570. https://doi.org/10.1029/2000GL011921

Jithin, A. K., Subeesh, M. P., Francis, P. A., \& Ramakrishna, S. S. (2020). Intensification of tidally generated internal waves in the north-central Bay of Bengal. Scientific Reports, 10(1), 6059. https://doi.org/10.1038/s41598-020-62679-4

Kelly, S. M. (2016). The vertical mode decomposition of surface and internal tides in the presence of a free surface and arbitrary topography. Journal of Physical Oceanography, 46(12), 3777-3788. https://doi.org/10.1175/jpo-d-16-0131.1

Kelly, S. M., Jones, N. L., Ivey, G. N., \& Lowe, R. J. (2014). Internal-tide spectroscopy and prediction in the Timor Sea. Journal of Physical Oceanography, 45(1), 64-83. https://doi.org/10.1175/JPO-D-14-0007.1

Kelly, S. M., \& Nash, J. D. (2010). Internal-tide generation and destruction by shoaling internal tides. Geophysical Research Letters, 37, L23611. https://doi.org/10.1029/2010GL045598

Kelly, S. M., Nash, J. D., \& Kunze, E. (2010). Internal-tide energy over topography. Journal of Geophysical Research, 115, C06014. https:// doi.org/10.1029/2009JC005618

Kelly, S. M., Waterhouse, A. F., \& Savage, A. C. (2021). Global dynamics of the stationary M2 mode-1 internal tide. Geophysical Research Letters, 48, e2020GL091692. https://doi.org/10.1029/2020GL091692

Kunze, E., Rosenfeld, L. K., Carter, G. S., \& Gregg, M. C. (2002). Internal waves in Monterey submarine canyon. Journal of Physical Oceanography, 32(6), 1890-1913. https://doi.org/10.1175/1520-0485(2002)032<1890:iwimsc >2.0.co;2

Matte, P., Jay, D. A., \& Zaron, E. D. (2013). Adaptation of classical tidal harmonic analysis to nonstationary tides, with application to river tides. Journal of Atmospheric and Oceanic Technology, 30(3), 569-589. https://doi.org/10.1175/JTECH-D-12-00016.1

Mellor, G., \& Yamada, T. (1982). Development of a turbulence closure model for geophysical fluid problems. Reviews of Geophysics, 20(4), 851-875. https://doi.org/10.1029/RG020i004p00851

Meyers, G., Bailey, R. J., \& Worby, A. P. (1995). Geostrophic transport of Indonesian throughflow. Deep-Sea Research Part I, 42(7), 11631174. https://doi.org/10.1016/0967-0637(95)00037-7

Morrow, R., Fu, L. L., Ardhuin, F., Benkiran, M., Chapron, B., Cosme, E., et al. (2019). Global observations of fine-scale ocean surface topography with the Surface Water and Ocean Topography (SWOT) mission. Frontiers in Marine Science, 6, 232. https://doi.org/10.3389/fmars.2019.00232

Munk, W. H., \& Cartwright, D. (1966). Tidal spectroscopy and prediction. Philosophical Transactions of the Royal Society of London, 259(1105), 533-581. https://doi.org/10.2307/j.ctt211qv60.7

Murphy, A. (1988). Skill scores based on the mean square error and their relationships to the correlation coefficient. Monthly Weather Review, 16(12), 2417-2424. https://doi.org/10.1175/1520-0493(1988)116<2417:ssbotm >2.0.co;2

Nash, J. D., Kelly, S. M., Shroyer, E. L., Moum, J. N., \& Duda, T. F. (2012a). The unpredictable nature of internal tides on continental shelves. Journal of Physical Oceanography, 42(11), 1981-2000. https://doi.org/10.1175/JPO-D-12-028.1

Nash, J. D., Shroyer, E. L., Kelly, S. M., Inall, M., Duda, T. F., Levine, M., et al. (2012b). Are any coastal internal tides predictable? Oceanography, 25(2), 80-95. https://doi.org/10.5670/oceanog.2012.44

Nelson, A. D., Arbic, B. K., Zaron, E. D., Savage, A. C., Richman, J. G., Buijsman, M. C., \& Shriver, J. F. (2019). Toward realistic nonstationarity of semidiurnal baroclinic tides in a hydrodynamic model. Journal of Geophysical Research: Oceans, 124, 6632-6642. https://doi. org/10.1029/2018JC014737

Pickering, A., Alford, M., Nash, J., Rainville, L., Buijsman, M., Ko, D. S., \& Lim, B. (2015). Structure and variability of internal tides in luzon strait. Journal of Physical Oceanography, 45(6), 1574-1594. https://doi.org/10.1175/JPO-D-14-0250.1 
Ponte, A. L., \& Klein, P. (2015). Incoherent signature of internal tides on sea level in idealized numerical simulations. Geophysical Research Letters, 42, 1520-1526. https://doi.org/10.1002/2014GL062583

Rainville, L., Johnston, T. M., Carter, G. S., Merrifield, M. A., Pinkel, R., Worcester, P. F., \& Dushaw, B. D. (2010). Interference pattern and propagation of the M2 internal tide south of the Hawaiian Ridge. Journal of Physical Oceanography, 40(2), 311-325. https://doi. org/10.1175/2009JPO4256.1

Rainville, L., \& Pinkel, R. (2006). Propagation of low-mode internal waves through the ocean. Journal of Physical Oceanography, 36(6), 1220-1236. https://doi.org/10.1175/JPO2889.1

Ray, R. D., \& Zaron, E. D. (2011). Non-stationary internal tides observed with satellite altimetry. Geophysical Research Letters, 38 , L17609. https://doi.org/10.1029/2011GL048617

Rayson, M. D., Gross, E. S., \& Fringer, O. B. (2015). Modeling the tidal and sub-tidal hydrodynamics in a shallow, micro-tidal estuary. Ocean Modelling, 89, 29-44. https://doi.org/10.1016/j.ocemod.2015.02.002

Rayson, M. D., Ivey, G., Jones, N., \& Fringer, O. (2018). Resolving high-frequency internal waves generated at an isolated coral atoll using an unstructured grid ocean model. Ocean Modelling, 122, 67-84. https://doi.org/10.1016/j.ocemod.2017.12.007

Rayson, M. D., Jones, N., \& Ivey, G. (2012). Temporal variability of the standing internal tide in the Browse Basin, Western Australia. Journal of Geophysical Research, 117, C06013. https://doi.org/10.1029/2011JC007523

Robertson, R., \& Ffield, A. (2008). Baroclinic tides in the Indonesian seas: Tidal fields and comparisons to observations. Journal of Geophysical Research, 113, C07031. https://doi.org/10.1029/2007JC004677

Sarkar, D., Osborne, M. A., \& Adcock, T. A. (2018). Prediction of tidal currents using Bayesian machine learning. Ocean Engineering, 158, 221-231. https://doi.org/10.1016/j.oceaneng.2018.03.007

Savage, A. C., Arbic, B. K., Richman, J. G., Shriver, J. F., Alford, M. H., Buijsman, M. C., et al. (2017). Frequency content of sea surface height variability from internal gravity waves to mesoscale eddies. Journal of Geophysical Research: Oceans, 122, 2519-2538. https://doi. org/10.1002/2016JC012331

Savage, A. C., Waterhouse, A. F., \& Kelly, S. M. (2020). Internal tide nonstationarity and wave-mesoscale interactions in the Tasman Sea. Journal of Physical Oceanography, 50(10), 2931-2951. https://doi.org/10.1175/JPO-D-19-0283.1

Shriver, J. F., Richman, J. G., \& Arbic, B. K. (2014). How stationary are the internal tides in a high-resolution global ocean circulation model? Journal of Geophysical Research: Oceans, 119, 2769-2787. https://doi.org/10.1002/2013JC009423

Stammer, D., Ray, R. D., Andersen, O. B., Arbic, B. K., Bosch, W., Carrère, L., et al. (2014). Accuracy assessment of global barotropic ocean tide models. Reviews of Geophysics, 52, 243-282. https://doi.org/10.1002/2014RG000450

Torres, H. S., Klein, P., Menemenlis, D., Qiu, B., Su, Z., Wang, J., et al. (2018). Partitioning ocean motions into balanced motions and internal gravity waves: A modeling study in anticipation of future space missions. Journal of Geophysical Research: Oceans, 123, 8084-8105. https://doi.org/10.1029/2018JC014438

Van Haren, H. (2004). Incoherent internal tidal currents in the deep ocean. Ocean Dynamics, 54(1), 66-76. https://doi.org/10.1007/ s10236-003-0083-2

Wunsch, C. (2013). Baroclinic motions and energetics as measured by altimeters. Journal of Atmospheric and Oceanic Technology, 30(1), 140-150. https://doi.org/10.1175/JTECH-D-12-00035.1

Zaron, E. D. (2017). Mapping the nonstationary internal tide with satellite altimetry. Journal of Geophysical Research: Oceans, $122,539-554$. https://doi.org/10.1002/2016JC012487

Zaron, E. D. (2019). Baroclinic tidal sea level from exact-repeat mission altimetry. Journal of Physical Oceanography, 49(1), 193-210. https://doi.org/10.1175/JPO-D-18-0127.1

Zaron, E. D., \& Egbert, G. D. (2014). Time-variable refraction of the internal tide at the Hawaiian Ridge. Journal of Physical Oceanography, 44(2), 538-557. https://doi.org/10.1175/JPO-D-12-0238.1

Zhao, Z., Alford, M. H., Girton, J. B., Rainville, L., \& Simmons, H. L. (2016). Global observations of open-ocean mode-1 internal tides. Journal of Physical Oceanography, 46(6), 1657-1684. https://doi.org/10.1175/JPO-D-15-0105.1 